Andreas Vasilache

\title{
Exekutive Gouvernementalität und die Verwaltungsförmigkeit internationaler Politik
}

\begin{abstract}
Die Abwendung Foucaults vom Staat sowie von staatstheoretischen Konzepten hat die Fruchtbarmachung seines Denkens in den Internationalen Beziehungen erschwert und begrenzt. In diesem Artikel soll ein Beitrag zur Konkretisierung des analytischen wie kritischen Potenzials der gouvernementalitätstheoretischen Perspektive für die Analyse grenzüberschreitender Politik geleistet werden, indem die Anschlussfähigkeit des Gouvernementalitätsansatzes über einen engeren Foucault-Diskurs hinaus diskutiert und ausgewiesen wird. In einem ersten Schritt wird dargelegt, dass der Foucaultsche Gouvernementalitätsansatz auf die staatstheoretische Gewaltenteilungslogik rekurriert und mit der Struktur der exekutiven Gewalt korrespondiert. Im Anschluss daran wird der mögliche Beitrag einer staatstheoretisch rückbezogenen Foucaultschen Perspektive zum Ausweis einer verwaltungslogischen (Um-)Formung grenzüberschreitender Politik ausgewiesen und damit das empirische Feld umrissen, in dem ein solcher Ansatz fruchtbar gemacht werden kann. Schließlich wird im dritten Teil dieses Feld durch Hinweise auf empirische Phänomene und politische Trends aus dem Sachbereich der Sicherheit konkretisiert und an einigen explorativen Beispielen das Problem des verwaltungslogischen mainstreaming der Politik exemplifiziert.
\end{abstract}

\section{Einleitung}

Dass die Diskussion Foucaultscher Ansätze in den Internationalen Beziehungen (IB) nicht im gleichen Maße fortgeschritten ist wie in anderen politikwissenschaftlichen Subdisziplinen - und m.E. wohl noch gar nicht ausgemacht ist, inwieweit die Arbeiten Michel Foucaults in den IB einen fruchtbaren Beitrag zu leisten in der Lage sind -, zeigt sich an den tentativen und insgesamt betrachtet kumulativen Bezugnahmen zum Foucaultschen Denken. ${ }^{1}$ So stellen Mathias Albert und Peter Lenco (2008: 265; vgl. Diez 2008: 267) das grundsätzliche Potenzial Foucaults zur Diskussion, indem sie die "variety in the adoptation, uses, and appropriations of his work « hervorheben. Die noch junge Rezeptionsgeschichte in den IB zeigt sich auch darin, dass oft überaus breit und allgemein von der Ausweitung Foucaultscher Ansätze »to international affairs « (Hindess 2008: 269) oder noch allgemeiner »the international« (Hindess 2008: 268) ${ }^{2}$ oder »the global« (Merlingen 2008: 273; vgl. Lipschutz/Rowe 2005: 14) gesprochen oder affirmativ global governmentality als Wechselbegriff von global

1 Für präzise Kritik sowie hilfreiche Anregungen, Hinweise und Verbesserungsvorschläge möchte ich Mathias Albert und Mark Arenhövel, den anonymen Gutachterinnen und Gutachtern der ZIB sowie der ZIB-Redaktion meinen Dank aussprechen.

2 Vgl. i.d.S. auch Joseph (2010: 223); Neumann/Sending (2007: 677, 694). 
governance verwendet wird. ${ }^{3}$ Es ist zwar festzustellen, dass " governmentality as an analysis of representations of social problems, the means to remedy them and their effects on the construction of subjectivity has informed a series of approaches in International Relations« (Aradau/Van Munster 2007: 91). Doch auch in diesem Zitat wird letztlich die punktuelle und tentative Bezugnahme auf Foucault hervorgehoben. Zudem liegt ein Interessensschwerpunkt der gouvernementalitätstheoretischen Beiträge auf Phänomenen der biopolitischen Subjektkonstituierung (Aradau 2004; de Larrinaga/Doucet 2008; 2010a; Dillon 2007; Kiersey 2010), wobei sie bisweilen einen nur indirekten Bezug zu Foucaults Arbeiten aufweisen (Di Muzio 2008; Ferguson/Gupta 2002; Aradau/Van Munster 2007). In diesem Sinne sind auch die u.a. auf Foucault bezogenen sicherheitstheoretischen Überlegungen der Paris School vornehmlich auf Fragen biopolitischer Subjektivierung, Normalisierung und Exklusion gerichtet. ${ }^{4}$ Solche Ansätze sind wichtig und lehrreich, aber in den IB und den Europastudien alleine nicht hinreichend, da Institutionen betreffende oder gar staatstheoretische Fragen in systematischer Hinsicht kaum eine Rolle spielen oder sogar grundsätzlich verworfen werden (Bigo 2008: 102, 106, 113; Bigo et al. 2006: Kap. 7.4-7.6). Hierdurch wird die Anschlussfähigkeit Foucaults an Forschungen außerhalb eines engeren subjektologischen und biopolitischen Paradigmas erschwert. ${ }^{5}$

Dass die Frage nach möglichen Adaptionen Foucaults noch nicht hinreichend geklärt ist, mag ganz allgemein auch daran liegen, dass konstruktivistische und poststrukturalistisch inspirierte Ansätze in den IB sich insgesamt schwerpunktmäßig auf die Einsicht konzentrieren, dass politische Realitäten im weitesten Sinne keine objektiven Gegebenheiten, sondern immer auch ideelle und soziale Konstrukte sind und daher die Untersuchung gegenstandskonstituierender Diskurse ins Zentrum der Analyse rücken. ${ }^{6}$ Diese konstruktivistische Grundeinsicht (vgl. Kerchner 2006: 149-151) aufzugreifen und für die empirische Erforschung der Konstruktion sozialer Tatbestände, Ideen, Identitäten, Interessen etc. in der internationalen Politik nutzbar zu machen, ist dabei zwar überaus erhellend, stellt aber eine recht begrenzte Adaption der (umfang-)reichen politiktheoretischen Diskussion poststrukturalistischer Ansätze in den IB dar.

Neben dieser allgemeinen Tendenz spielen auch speziellere Gründe für die zurückhaltende und eher punktuelle Fruchtbarmachung der politischen Theorie Fou-

3 Hiervor sind auch Lipschutz/Rowe (2005: 7) nicht ganz gefeit, wobei sie allerdings später das Konzept der Gouvernementalität konkretisieren und die Gleichsetzung mit dem governance-Konzept implizit relativieren (Lipschutz/Rowe 2005: 13-15, 55-57).

4 Vgl. Bigo (2008: 103-105); C.A.S.E. Collective (2006: 456-458); Bigo et al. (2006: Kap. 6.3, 7.1-7.3, 8.4).

5 Jessop $(2007$; 2011) mahnt eine explizit staatstheoretische Beschäftigung mit Foucault an, belässt es aber bei der (wichtigen) Feststellung, dass man auch aus einer Foucaultschen Perspektive den Staat berücksichtigen könne und müsse sowie bei dem Schlagwort einer »idea of government as strategic codification of power relations « (2007: 39). Jessop bleibt also bei der Forderung einer staatstheoretischen Diskussion stehen, ohne sich selbst staatstheoretisch mit Foucault zu befassen oder hierfür einen Vorschlag zu unterbreiten.

6 Vgl. noch immer Wendt (2004) sowie Zehfuss (2002), Diez (1999), Wiener (2003), Risse (2004: 160-166), Wæver (2004: 197-202) und für die politische Theorie Kerchner/Schneider (2006: 9-10). 
caults in den IB eine Rolle. So waren und sind in der politiktheoretischen Foucaultrezeption insgesamt und der Rezeption seiner Theorie der Gouvernementalität im Besonderen die Bezugnamen zur internationalen Politik kaum ausgeprägt. ${ }^{7}$ Der Grund hierfür - und mithin der zweite konkretere Grund für die verhaltene Rezeption Foucaults in den IB - könnte nicht nur am beinahe vollständigen Fehlen jedweder Bezugnahmen zum Internationalen in Foucaults Texten selbst liegen, ${ }^{8}$ sondern in Foucaults theoretischer Abwendung sowohl vom Staat als auch von der Idee institutionalisierter und formal organisierter politischer Macht. Da Foucault nicht lediglich den Staat für ein bedeutungsmäßig nachgelagertes Phänomen hält, sondern seine Skepsis institutionalisierten politischen Entitäten - wie auch dem positiv gesetzten Recht - überhaupt gilt, macht er es nicht nur (neo-) realistischen bzw. etatistischen Denkströmungen in den IB schwer, sich auf ihn zu beziehen, ${ }^{9}$ sondern erschwert auch institutionalistischen, liberalen oder global governance-Ansätzen eine Berufung auf seine politische Theorie.

Ich denke nun aber, dass die immer wieder als defizitär kritisierte Unterberücksichtigung politischer Institutionen und des Staates bei Foucault sowie in den governmentality studies ${ }^{10}$ in einem wesentlichen Aspekt, nämlich dem der exekutiven Inklination der Gouvernementalität, staatstheoretisch aufgefangen werden kann - und dass dies schließlich eine Fruchtbarmachung seines Ansatzes für die Erforschung grenzüberschreitender Politik ${ }^{11}$ erlaubt. Es ist daher ein Mittelweg auszuweisen, der einen staatstheoretisch-institutionellen Blick mit der Gouvernementalitätsperspektive zusammenzuführen verspricht. Während die Adaptionen Foucaults in den IB sich meist der Foucaultschen Theorie von konkreten empirischen Gegenständen und Interessen angenähert haben, scheint es mir dabei sinnvoll, in umgekehrter Richtung zu verfahren. Gerade weil das Foucaultsche Denken selbst eine umstandslose Anwendung in den IB erschwert, ist es, gleichsam in ergänzender Absicht, geboten und plausibel, die Argumentation aus einer theoretischen Diskussion zu entfalten und anschließend auf empirische Phänomene zu beziehen, deren Zusammenhänge und Relevanz für die grenzüberschreitende Politik z.T. erst in einer solchen theoretischen Optik sichtbar werden. Dabei können auch bislang unzureichend verbundene Diskurse in den IB und der politischen Theorie - ganz im Sinne aktueller Debatten zu

7 Vgl. z.B. Hindess (1996), Barry et al. (1996), Burchell et al. (1991), Dean (1999), Krasmann/Volkmer (2007), Bröckling et al. (2011) sowie - mit einem ausdrücklichen Interesse an Foucaults Staatsverständnis - Heidenreich (2011a).

8 Vgl. auch Lipschutz/Rowe (2005: 15, 55); Caldwell (2007: 111, 115); Neumann/Sending (2007: 700).

9 Vgl. Neumann/Sending (2007) für einen interessanten Versuch, Hans J. Morgenthaus Konzeptualisierung des Internationalen mit Rekurs auf Foucaults Gouvernementalitätsansatz zu aktualisieren.

10 Für diese Kritik vgl. Lemke (2007: 54, 59), Opitz (2008: 215-220), Joseph (2010: 241-243), Vasilache (2007: 216-217; 2009) sowie Jessop (2011: 57, 60-63, 69-70), der betont, dass die Zurückweisung des Staates in den governmentality studies Foucaults Interesse an Makrostrukturen nicht entspräche.

11 Ich verwende den Ausdruck der grenzüberschreitenden Politik als zusammenfassenden Begriff, der inter-, trans- und supranationale Politik umfasst. 
Internationaler Politischer Theorie (IPT) ${ }^{12}$ - miteinander ins Gespräch gebracht werden.

Das vornehmliche Ziel dieses Beitrags lässt sich im Hinblick auf die dargestellten Problemlagen somit in mehrere miteinander zusammenhängende Punkte auffächern. So kann die staatstheoretische Rückbeziehung der Gouvernementalitätstheorie erstens einen Beitrag zur Theoriediskussion in zwei Bereichen leisten. Dabei wird ein Vorschlag präsentiert, der einerseits Foucaults problematische Unterberücksichtigung von politischen Institutionen und Entitäten aufzubrechen erlaubt, dabei den Staat nicht lediglich in Sammelbegriffen (wie Souveränität, souveräne Macht, Biopolitik) thematisiert, ${ }^{13}$ und andererseits den analytischen Rahmen der Betrachtung von Regierungshandeln erweitern kann. Zweitens sollen die folgenden Überlegungen die existierenden Bezugnahmen auf poststrukturalistische Ansätze in der Theorie der IB ergänzen, indem ein Beitrag zur Konkretisierung des analytischen wie kritischen Potenzials der gouvernementalitätstheoretischen Perspektive Foucaults in der Analyse grenzüberschreitender Politik geleistet wird, der auch über die Theoriegemeinde erklärter Foucault-Anhängerinnen und -Anhänger hinaus anschlussfähig zu sein verspricht. Obgleich die Arbeit mit poststrukturalistischen Ansätzen dies nahe zu legen scheint, wird also ausdrücklich keine grand theory im Sinne der These einer gouvernementalen »Regierung der Menschheit« (Caldwell 2007: 110) und keine makroperspektivische Diskussion im Sinne einer Betrachtung des »International as Governmentality (Neumann/Sening 2007: 690, meine Hervorh.) bzw. von »Globalization as Governmentality« (Larner/Walters 2004: 495, meine Hervorh.) angestrebt. Vielmehr soll die Diskussion einerseits auf die Beziehung zwischen Foucaultscher Gouvernementalität und exekutiver Gewalt im klassischen staatstheoretischen Sinne sowie andererseits auf die Möglichkeiten der Operationalisierung einer solchen konzeptuellen Verbindung in den IB konzentriert und mithin begrenzt werden. Dabei wird insbesondere der Beitrag einer solchen Perspektive zum Verständnis einer verwaltungslogischen (Um-)Formung internationaler Politik dargelegt. Schließlich soll drittens dieser theoretische Rahmen durch Hinweise auf ausgewählte empirische Beispiele und Trends aus dem Sachbereich der Sicherheit konkretisiert und an ihnen das Problem des verwaltungslogischen mainstreaming der Politik exemplifiziert werden. Hier wird explorativ sowohl zu zeigen sein, dass und wie Fragen der Sicherheit gouvernementalisiert werden, als auch, dass gerade die exekutive Inklination der Gouvernementalität die Berücksichtigung klassischer, gleichsam staats- und militärfokussierter Sicherheitslogiken und -politiken zulässt.

In diesem Sinne möchte ich in einem ersten Schritt begründen, dass der Foucaultsche Gouvernementalitätsansatz auf Aspekte der klassischen Gewaltenteilung und

12 Vgl. hierzu das Symposium zur IPT in der ZIB (2010).

13 Den Staat als unspezifizierten Sammelbegriff einzuführen, entspricht Foucaults Umgang mit dem Staatsbegriff und wird in aktuellen Bezugnahmen auf seine Theorie gespiegelt, was - je nach theoretischer und/oder empirischer Interessenslage - durchaus plausibel sein kann. Vgl. Di Muzio (2008), Ferguson/Gupta (2002: 989), Dean (2007: Kap. 6), Vasilache (2009), de Larrinaga/Doucet (2008; 2010b) sowie Heng/McDonagh (2008: 573), die zugleich allerdings betonen, dass die Frage der agency der Aufmerksamkeit bedürfe (2008: 570). 
dabei insbesondere auf die Handlungslogik der exekutiven Gewalt rekurriert bzw. rückbezogen ist. So wird zu zeigen sein, dass die Gouvernementalität Foucaults durchaus staatstheoretisch anschlussfähig ist, wobei das staatstheoretische Raster wiederum eine gouvernementale Transformation und Erweiterung erfährt. Im Anschluss daran soll aus einer theoretischen Diskussion heraus das empirische Feld umrissen werden, in dem eine staatstheoretisch geerdete gouvernementalitätstheoretische Perspektive fruchtbar gemacht werden kann. Schließlich ist im dritten Teil die Anwendbarkeit einer solchen Optik zu exemplifizieren. Anhand der Diskussion einiger empirischer Beispiele soll hier die verwaltungsförmige Rationalisierung im Gegenstandsbereich der grenzüberschreitenden Sicherheit veranschaulicht werden.

\section{Die staatstheoretische Anschlussfähigkeit Foucaults}

Foucault in die Nähe staatstheoretischer Betrachtungen zu rücken, scheint bestenfalls verwegen und rechtfertigungsbedürftig - wenn nicht sogar abwegig. Hatte sich Foucault nicht von einer juridisch-institutionellen Machtvorstellung und der staatstheoretischen Konzeptualisierung von Souveränität (vgl. Foucault 1975; 1976; 1978a; 1998) bzw. von der Besessenheit (Foucault 1978b: 38) von der Souveränität in der politischen Theorie losgesagt? In der Tat erkennt Foucault in der Machtkonzeption, die »um das Problem der Souveränität, also des Gesetzes, des Verbots herum konstruiert ist « (1978b: 38), einen anachronistischen Machttypus, der für die moderne Politik weitgehend unerheblich sei. Die Vorstellung eines klar lokalisierbaren, verbietenden Machtzentrums und einer hierarchischen sowie juridischen Funktionsweise der Macht sei vielmehr selbst noch als Machttaktik anzusehen, die ihre tatsächliche Arbeitsweise kaschiere (Foucault 1998: 48-49, 172-173) - so dass Didier Bigo zu dem Schluss kommt, dass aus Foucaults Perspektive »Hobbes [... ] a liar« (Bigo 2008: 106) sei. Gegen eine juridisch-souveräne Perzeption von Macht und Herrschaft stellt Foucault ein wesenhaft flexibles, unlokalisierbares und produktives Machtverständnis, das er definiert als die »Vielfältigkeit von Kräfteverhältnissen, die ein Gebiet bevölkern und organisieren« (Foucault 1998: 113). Im Gegensatz zu einer konzeptionellen Fassung der Macht in übergeordneten Begriffen wie Staat, Souveränität, binär arbeitender Legalität und schließlich Durchsetzung macht Foucault die Notwendigkeit einer Mikroanalytik der Macht geltend, die sich - wie Clemens Kammler (1986: 182) hervorhebt - auf die Faktizität von Machttechniken und folglich auf die Variabilität und Heterogenität der Macht, gleichsam auf ihre funktionale Mikroebene, konzentriert. Die Begriffe Recht, Souveränität und Staat werden von Foucault daher zumeist als Wechselbegriffe bzw. im Sinne eines umfassenden theoretischen Rahmens verwendet, von dem sich abzugrenzen sein Ziel ist. ${ }^{14}$ So identifiziert er schließlich das rechts-, souveränitäts- und staatszentrierte Denken in der politischen Theorie

14 Dean (2007: 134) betont, dass es sich bei dieser Abgrenzung um »a recurrent move in Foucault's work« handelt. 
als eine Reminiszenz monarchischer Vorstellungen und fordert, dass die politische Theorie endlich »dem König den Kopf abschlagen« (Foucault 1978b: 38) müsse.

In seiner späten Theorie der Gouvernementalität hat sich Foucault letztlich aber doch mit dem Staat und der Frage der Souveränität auseinandergesetzt. Dabei stellt er zwar weiterhin eine »Überbewertung des Staatsproblems« (Foucault 2004a: 163) fest, betont indes zugleich, dass das »Problem der Souveränität [...] nicht eliminiert« (Foucault 2004a: 161), sondern »im Gegenteil akuter denn je geworden« (Foucault 2004a: 161) sei. Dass es sich hier nicht um einen Widerspruch handelt, resultiert aus dem Verhältnis, das Foucault zufolge zwischen staatlicher Souveränität und gouvernementaler Regierungskunst besteht. Im Ausweis dieses Verhältnisses liegt der wesentliche Punkt, gleichsam der Clou seiner Argumentation begründet. Denn so kehrt Foucault das Verhältnis zwischen dem Staat und der Souveränität einerseits und der auf einer Mikrophysik der Machttechniken basierenden gouvernementalen Regierungskunst andererseits um. Er hebt hervor, dass es nicht die souveränen rechtsförmigen Institutionen seien, von denen die Machtanalyse ihren Ausgang nehmen solle, sondern dass der umgekehrte Weg angemessen sei und man Institutionen erst über die Analyse der Machtverhältnisse verstehen könne (Foucault 1994: 257; 2000a: 62). Nicht der souveräne Staat ist für Foucault ursprünglich für die Form und Ausübung politischer Macht, sondern umgekehrt sei der Staat »nichts anderes als der bewegliche Effekt eines Regimes vielfältiger Gouvernementalität« (2000b: 70; vgl. 2004a: 160). Um der Aktualität der Staatsthematik Rechnung zu tragen und dabei zugleich ihrer Überschätzung Einhalt zu gebieten, müsse man sich von der Frage einer vermeintlichen »Verstaatlichung der Gesellschaft« (Foucault 2004a: 163) ab- und der »Gouvernementalisierung des Staates« (Foucault 2004a: 163, Hervorh. dort) zuwenden. Der wesentliche Kritikpunkt Foucaults an der staats- und souveränitätszentrierten politischen Theorie besteht demnach darin, dass diese als Ausgangspunkt politischer Macht setze, was lediglich ihre Konsequenz sei.

Wenn Foucault nun die Staatstheorie gleichsam »against the Grain « (Amoore 2008: 274) liest, dann folgt hieraus zwar eine bedeutende perspektivische Verschiebung in der Analyse politischer Macht. So kann die Machtanalytik nicht mehr beim Begriff des souveränen Rechts stehenbleiben, sondern muss die vielfältigen Strategien, Taktiken, Ziele, Maßnahmen und Formen des Regierens in den Blick nehmen. ${ }^{15}$ Zugleich ist aber mit der Umkehrung des Verhältnisses von staatlicher Souveränität und gouvernementaler Macht nun doch eine positive Verbindung von Machttheorie einerseits

15 Dies ist auch der Grund, weshalb ich - im Unterschied zu de Larrinaga/Doucet (2008: 520-521) - in Giorgio Agambens Homos Sacer-Konzept (2002) keine Verbindung zwischen souverän-repressiver und diskursiv-produktiver Macht und damit keine staatstheoretische Fundierung des Foucaultschen Programms erkennen kann. Die von Agamben ausgewiesene totale souveräne Verfügbarkeit über das nackte Leben des Homo Sacer lässt von der diskursiven Produktion von Fürsorgesubjekten in der Foucaultschen Normalisierungsmacht letztlich nichts mehr übrig. Vgl. Dean (2007: 146-148), der Agamben und Foucault hinsichtlich ihrer biopolitischen Konzepte gemeinsam diskutiert, allerdings nicht zu einer Zusammenführung gelangt. Auch Bigo, prominenter Vertreter der Paris School, liest Agamben gegen Foucault. Dies allerdings resultiert aus der Ablehnung einer staatstheoretischen Rückbeziehung Foucaults überhaupt, die ich nicht teile (vgl. Bigo 2008: 110, 113). 
und Staatlichkeit und Souveränität andererseits gegeben. In den machtanalytischen Texten Foucaults aus den 1970er Jahren standen Macht und Souveränität noch in einem Ausschlussverhältnis zueinander (Foucault 1975; 1976; 1978a; 1978b). Die Begriffe des Rechts, der Staatlichkeit und der Souveränität dienten, so sie denn Erwähnung fanden, als Stichwortgeber für die Kritik an vermeintlich überholten Vorstellungen einer negativ-repressiven Machtform und damit als negative Korrelate eines diskursiv-produktiven Machtbegriffs. ${ }^{16}$ Mit der Aufwertung des Staates zu einem Produkt gouvernementaler Macht überwindet Foucault das vormalige Ausschlussverhältnis zugunsten einer Beziehung von Ursache und Wirkung - selbst wenn Ursache und Wirkung gegenüber der klassischen staatstheoretischen Logik umgekehrt sind. So lässt sich erstens festhalten, dass Foucault mit der Analyse moderner Gouvernementalität gleichsam die bei ihm zuvor nur reziprok-exkludierend gefassten Phänomene der Macht und der Souveränität bzw. des Rechts verbindet.

Dass der gouvernementalitätstheoretische Ansatz Foucaults durchaus mit einer staatstheoretischen Perspektive zusammengeführt werden kann, zeigt sich neben diesem ersten, eher allgemeinen und theoriesystematischen Argument allerdings zweitens, wenn man das Konzept der Gouvernementalität in seiner Detailstruktur betrachtet:

»Es geht also [...] nicht darum, den Menschen ein Gesetz aufzuerlegen, es geht darum, Dinge anzuordnen, das heißt eher Taktiken als Gesetze [...] einzusetzen und es durch eine bestimmte Anzahl von Mittel so einzurichten, daß dieses oder jenes Ziel erreicht werden kann« (Foucault 2004a: 150).

»Mit dem Wort >Gouvernementalität< möchte ich drei Dinge sagen. Ich verstehe unter >Gouvernementalität` die aus den Institutionen, den Vorgängen, Analysen und Reflexionen, den Berechnungen und den Taktiken gebildete Gesamtheit, welche es erlauben, diese [...] Form der Macht auszuüben, die als Hauptzielscheibe die Bevölkerung, als wichtigste Wissensform die politische Ökonomie und als wesentliches technisches Instrument die Sicherheitsdispositive hat. Zweitens verstehe ich unter >Gouvernementalität « die Tendenz oder die Kraftlinie, die im gesamten Abendland [...] zur Vorrangstellung dieses Machttypus geführt hat, den man [...] die >Regierung nennen kann: Souveränität, Disziplin, und die $[\ldots]$ die Entwicklung einer ganzen Serie spezifischer Regierungsapparate [sowie] [...] von Wissensarten nach sich gezogen hat. Schließlich denke ich, daß man unter >Gouvernementalitätく den Vorgang oder vielmehr das Ergebnis des Vorgangs verstehen sollte, durch den der mittelalterliche Staat der Gerichtsbarkeit, der im 15. und 16. Jahrhundert zum Verwaltungsstaat wurde, sich nach und nach gouvernementalisiert hat « (Foucault 2004a: 162-163, Hervorh. dort).

Der letztgenannte Punkt ist freilich tautologisch. Dennoch ist auch dieser aufschlussreich, um sowohl die Anschlussfähigkeit des Foucaultschen Gouvernementalitätsansatzes an eine staatstheoretische Perspektive als auch das Spezifische der Gouvernementalität zu demonstrieren. So tritt zum einen erneut die Differenz zutage, die Foucault zwischen der Souveränität, dem Recht und dem Staate einerseits und der Gouvernementalität andererseits erkennt und die darin besteht, »daß es sicherlich nicht das Gesetz ist, durch das die Ziele des Regierens tatsächlich zu erreichen sind «

16 Diese Vernachlässigung juridisch-repressiver Machtformen bei Foucault ist vielfach und aus unterschiedlichen Blickwinkeln kritisiert worden, z.B. von Butler (2003: 52-67), Gehring (2004: 117-119), Fraser (2003: 250-251) sowie Vasilache (2007: 216-222). 
(Foucault 2004a: 150). Zum anderen aber wird deutlich, inwiefern und auf welcher Ebene das Konzept der Gouvernementalität einer staatstheoretischen Begrifflichkeit und Systematik entspricht. Denn wenn Foucault von der Regierungskunst, vom Regieren, schließlich von »allgemeine[r] Verwaltung « (Foucault 2004a: 147) und kurze Zeit später vom »Regierungsstaat« (Foucault 2004a: 164) spricht, dann liegt schon begrifflich eine besondere Nähe der Gouvernementalität zur exekutiven Gewalt des Staates, d.h. zum Regieren im engeren und tradierten staatstheoretischen Sinne des Begriffes nahe.

Dabei gilt es hervorzuheben, dass es sich um eine struktur- und funktionslogische Korrespondenz mit der staatstheoretischen Idee der Exekutive handelt, die weder auf eine konkrete Staatstheorie noch gar auf eine bestimmte empirische Realisierungsform der Gewaltenteilung, -trennung oder -verschränkung bezogen ist. Vielmehr betrifft sie die in der »politischen Methodologie des [... Staates« (Vasilache 2007: 337, vgl. 108-114, 182-186, 320-338) angelegte systematische Grenzziehung zwischen legislativem und exekutivem Aktivitätsbereich. Es ist eine Grenze, die die »Verteilung von Zuständigkeiten« und nicht die konkrete (macht-)politische »Teilung der Gewalt« (Jellinek 1966: 501) bestimmt.

Diese (idealtypische) Unterscheidung ist bereits der Idee des Rechts und damit dem Konzept des Verfassungs- und Rechtsstaats inhärent und resultiert daraus, dass das Gesetz aufgrund seiner Allgemeinheit in der Regel weder Individuen noch Einzelfragen in ihrer Partikularität direkt zu adressieren vermag. ${ }^{17}$ Jean-Jacques Rousseau bringt die epistemisch-kategoriale Systematik der Unterscheidung zwischen legislativer und exekutiver Tätigkeit auf den Punkt und betont, dass das Gesetz die Stimme aller sei, die sich immer nur an alle zugleich wenden könne, während die Anwendung der Gesetze stets ein partikularer Akt sei (Rousseau 1977: 28-29, 33-34, 35-38, 61-62, 135). Da die Verwaltung »nur aus einzelnen Akten besteht, die in keiner Weise in den Bereich des Gesetzes und folglich auch nicht in den des Souveräns fallen, dessen Akte alle nur Gesetze sein können« (Rousseau 1977: 61-62), bildet für Rousseau die konkretisierende Applizierung der Gesetze im Einzelfall sowie die Bearbeitung von gesetzlich nicht normierten Einzelfragen einen von der legislativen Souveränität kategorial unterschiedenen Aufgabenbereich. ${ }^{18}$ Auch Charles de Montesquieu, der die Einrichtung dreier Staatsgewalten begründet, erkennt in der Unterscheidung zwischen allgemeinem Willen und partikularer Implementierung eine wesentliche systematische Grenze zwischen legislativem und exekutivem Sachbereich (Montesquieu 1992: 28-31, 215-218). Diese systematische Unterscheidung tritt ebenso in John Lockes

17 Dieses kategoriale Scheidungsprinzip schließt - da wir es im politisch-gesellschaftlichen Bereich ja nicht mit mathematischen Gleichungen zu tun haben - in der Praxis nicht aus, dass sich legislative Organe bestimmte Einzelentscheidungen vorbehalten können. In der Regel handelt es sich um außergewöhnliche Entscheidungen mit großer (politischer oder symbolischer) Tragweite, wie beispielsweise Kriegserklärungen und Friedensschlüsse oder auch die Vergabe von Orden. Als Beispiel aus der jüngeren deutschen Geschichte nennt Lembcke (2010: 245) das Gesetz über die Verlegung des Parlaments- und Regierungssitzes von Bonn nach Berlin.

18 Dies ist im Übrigen auch der Grund dafür, dass sowohl Locke als auch Rousseau die Rechtsprechung als Teil der Exekutive ansehen. 
Gewaltenteilungskonzept sowie insbesondere in seinen Darlegungen zur prärogativen Kompetenz hervor (Locke 1966: $\S 136, \S 143-144, \S 153, \S 158$ ). Und selbst bei Thomas Hobbes, der - wie Rousseau auch - ausdrücklich die Möglichkeit einer praktischen Gewaltenteilung ausschließt, ist doch diese epistemisch-systematische Unterscheidung von legislativer und exekutiver Tätigkeit der von ihm hervorgehobenen Allgemeinheit des Gesetzes inhärent (Hobbes 1998: 222, vgl. 203-244). Dieser epistemisch-kategoriale Basisentwurf der Exekutive findet sich indes nicht nur im klassischen Kontraktualismus oder in dem auf ihn unmittelbar zurückgehenden Denken Carl Schmitts (1996: 53-55), sondern beispielsweise auch bei Georg Jellinek, der »Normsetzung und Erledigung einzelner bestimmter Aufgaben durch auf sie gerichtete individualisierte Tätigkeit« (Jellinek 1966: 610) unterscheidet und hervorhebt, dass »abstrakte Regeln [...] als solche nicht unmittelbar die Wirklichkeit beherrschen « (Jellinek 1966: 610, vgl. 610-618). Das auf das Einzelne und Partikulare gerichtete Interesse der Exekutive tritt auch deutlich in dem Ausweis der personalen und sachbezogenen Instrumentalität der Verwaltung bei Max Weber (1947: 149, 151-152) zutage. Und schließlich betont selbst Hermann Heller, dass »jede Konkretisierung des Gesetzes durch den [...] Verwaltungsbeamten eine folgenreiche interpositio auctoritatis bedeutet« (Heller 1971: 225, Hervorh. dort, vgl. 59-61, 117, 123 , 217, 225-226). ${ }^{19}$ Insgesamt wird deutlich, dass in all diesen staatstheoretischen Betrachtungen der systematische Bereich der exekutiven Tätigkeit durch diese epistemisch-kategoriale Grenzziehung zwischen allgemeiner und partikularer Geltung umrissen wird. Ohne dass sich die Theorie oder gar die Praxis der Gewaltenteilung hierin erschöpfen würde, ${ }^{20}$ kann gleichwohl festgestellt werden, dass die Differenz zwischen gesetzgebender und administrativer Staatstätigkeit in ihrem Kern immer auch eine systemlogische ist, die der Idee des Rechts selbst immanent ist.

Wenngleich Foucault eine Verabschiedung der Staatstheorie en gros beabsichtigt, weist nun sein Konzept der Gouvernementalität gewichtige Kongruenzen mit der Struktur, den Aufgaben und der Funktionslogik der exekutiven Gewalt im dargelegten staatstheoretischen Sinne auf. Es zeigt sich, dass die Foucaultsche Gouvernementalität vornehmlich die exekutive Staatstätigkeit betrifft bzw. sich auf der Ebene und in der Form der Verwaltung realisiert. So ergeben sich bei Betrachtung des Foucaultschen Gouvernementalitätsbegriffs sechs wesentliche Kongruenzen mit der staatstheoretischen Konzeptualisierung der exekutiven Gewalt:

19 Ohne dies hier vertiefen zu können, sei doch angemerkt, dass Heller sich vehement gegen die Zweiteilungslehre des Gesetzes (als materielles und formelles) ausspricht, die rechtsdogmatisch aus der Unterscheidung zwischen allgemeinem Recht und partikularer Anwendung hergeleitet wurde (Heller 1971: 214-216), und sich auch gegen die Vorstellung einer rein formell konkretisierenden Rechtsprechung und Verwaltung wendet (Heller 1971: 61, 114, 123, 225-226). Vgl. hierzu Lembcke (2010). Zu betonen ist gleichwohl, dass dies nicht der These widerspricht, dass sich die Exekutive in systematischer Hinsicht durch einen schwerpunktmäßig auf Einzelfragen bezogenen Blick auszeichnet. Hierdurch findet sich die auch inhaltlich relevante Rolle der exekutiven Tätigkeit keineswegs negiert.

20 Es soll und kann hier keinesfalls bestritten werden, dass die empirische Institutionalisierung und die Praxis der Gewaltenteilung maßgeblich von historischen, kulturellen, institutionellen, macht- und rechtspolitischen Bedingungen abhängen. 
- Erstens weisen sowohl die gouvernementale Macht bei Foucault als auch die exekutive Staatsgewalt einen Fokus im Bereich des Konkreten, des Einzelnen und des Partikularen und damit der Anwendung, Implementierung und Durchsetzung auf, was sie - wie in der staatstheoretischen Diskussion soeben dargelegt - von der auf das Allgemeine gerichteten und notwendig vom Partikularen abstrahierenden Logik des Rechts und der Gesetzgebung unterscheidet (vgl. Foucault 2004a: 488-489).

- Dieser Punkt hängt zweitens mit einer weiteren Kongruenz zusammen, die in der systematisch nachrangigen Rolle des Gesetzes für die Aufgabenstellung und Arbeitsweise sowohl der gouvernementalen Macht als auch der exekutiven Gewalt besteht. Diese Nachrangigkeit des Gesetzes bzw. diese Gesetzesferne resultiert im ersten Fall daraus, dass für Foucault das Gesetz lediglich eine Konsequenz von Machtbeziehungen ist (Foucault 2004a: 150) und folgt im zweiten Fall aus der fehlenden Gesetzgebungskompetenz der Regierung ${ }^{21}$ sowie ihrer besonderen Bedeutung im Hinblick auf gesetzlich nicht geregelte Fragen (Jellinek 1966: 617-618).

- Drittens teilt die gouvernementale Macht einen ordnungspolitischen Fokus mit der exekutiven Staatsgewalt (Foucault 2004a: 148-152).22

- Viertens werden die gouvernementale Macht und die exekutive Staatsgewalt durch einen Schwerpunkt auf die praktische Aufgabenbewältigung im Sinne der technokratischen Regulierung, Steuerung und des Managements (Foucault 2004a: 144-146, 488) sowie - damit zusammenhängend -

- fünftens durch ihren instrumentellen Erledigungs- und Problemlösungsbias geeint (Lemke 1997: 147; Weber 1947: 148-450, 210-212). Dabei vermag Foucaults Gouvernementalitätsansatz überdies auszuweisen, wie die diskursive »Problematisierung « (Foucault 1996: 178) bestimmter Phänomene funktioniert und durch welche diskursiven Strategien bestimmte Fragen und Sachverhalte als politisch belangvolle Gegenstände konstituiert und damit »zum Problem« (Foucault 1996: 178, Hervorh. dort) werden.

- Schließlich teilen gouvernementale Macht und exekutive Gewalt (letztere nicht zuletzt durch ihre polizeilichen und militärischen Apparate) sechstens eine besondere Aufmerksamkeit für Fragen der Sicherheit (Foucault 2004a: $162-163) .{ }^{23}$

21 So betont Rousseau, dass selbst ein zeitweise eingesetzter Diktator, der die Legislative aussetze und umfassende Vollmachten habe, doch nie legitim gesetzgebend tätig werden könne: »er kann alles machen mit Ausnahme von Gesetzen« (Rousseau 1977: 136).

22 Vgl. zum staatstheoretischen Konzept der Ordnungspolitik Anter (2003).

23 Vgl. hierzu auch Foucaults (2004a: 507) Hinweis auf die gouvernementale Notwendigkeit der Polizei. 
Dass Foucault bisweilen Verwaltung, Administration und Regieren weitgehend synonym verwendet (vgl. Foucault 2004a: 147, 506), ${ }^{24}$ deutet ebenfalls darauf hin, dass die gouvernementale Macht sich insbesondere auf die exekutive Staatsgewalt bezieht, oder besser gesagt: als auf sie bezogen angesehen werden muss. Denn wenngleich diese Begriffe in verschiedenen disziplinären und thematischen Kontexten keineswegs immer Wechselbegriffe sind, sind sie im Rahmen einer systematischen Gewaltenteilungslogik doch in der Tat weitgehend austauschbar. Aus einer solchen Perspektive zeichnen sich Verwaltung, Administration und Regieren/Regierung gleichermaßen durch einen Einzel- und Partikularfokus aus, wodurch sie von der Allgemeinheit der normsetzenden Gesetzgebung systematisch geschieden werden. ${ }^{25}$

Der grundlegende Unterschied zwischen der gouvernementalen Macht und der Konzeptualisierung der durchsetzenden Gewalt in der klassischen Gewaltenteilungstrias besteht nun darin, dass die Gouvernementalität über eine institutionalisierte Entität - wie es eine Regierung mitsamt Verwaltungsapparat darstellt - hinausgeht. Die Frage der satzungsmäßigen und institutionalisierten Organisation als geordneter und hierarchischer Akteur ist der gouvernementalen Macht nachgelagert. Während die Exekutive in der konstitutionellen Staatstheorie - idealtypisch - als formell institutionalisierter Akteur entworfen wird, ${ }^{26}$ kann aus einer gouvernementalitätstheoretischen Perspektive der Regierungsapparat nicht als Ursprung gouvernementaler Macht gelten, sondern (lediglich) als eine ihrer institutionalisierten Ausdruckformen (unter mehreren), mithin als ihre Konsequenz.

Wir haben es bei der Gouvernementalität letztlich mit einer allgemeinen Rationalität, gleichsam einer politischen Vernunft zu tun. Die »gouvernementale ratio, diese gouvernementale Vernunft« (Foucault 2004a: 415, Hervorh. dort) bezeichnet gemäß Lemke (1997: 147) »ein diskursives Feld, innerhalb dessen die Ausübung der Macht >rationalisiert « wird. [...] Eine politische Rationalität erlaubt also, ein Problem zu stellen und bietet bestimmte Lösungs- und Bearbeitungsstrategien für dieses Problem an«. Dabei ist die Gouvernementalität - ganz im Sinne der Foucaultschen Machtkonzeption - zugleich eine auf das Individuum wirkende Normalisierungsmacht, die nicht nur in der Regierung von Menschen, sondern ebenfalls in der Konstituierung von regierbaren Subjekten besteht: »These two levels of power can be distinguished analytically, though not in practice, firstly as the constitution of subjects and secondly as their regulation" (Simons 1995: 41). Kurzum, "governmentality produces populations that behave >normally«" (Lipschutz/Rowe 2005: 56). Die gouvernementale Macht umreißt und konstituiert als ihr Betätigungsfeld einen »aus den Menschen und den Dingen gebildete[n] Komplex« (Foucault 2004a: 146) mit all seinen Dynamiken und Interrelationen. Sie möchte »so etwas wie ein Lebens-, Existenz-, Arbeitsmilieu

24 Es ist allerdings hervorzuheben, dass Foucault in diesem Punkt begrifflich nicht einheitlich verfährt und an anderer Stelle Verwaltung und Regierung als unterschiedliche gouvernementale Logiken einführt, vgl. Foucault (2004a: 164) sowie für verschiedene gouvernementale Rationalitäten Kerchner (2006: 154).

25 Vgl. Foucault (2004a: 488-489) sowie Vasilache (2007: 151-158).

26 Vgl. Locke (1966: § 136, § 143-144, § 153, § 158); Rousseau (1977: 28-29, 33-34, 35-38, 61-62, 135-136); Montesquieu (1992: 28-31, 215-218). 
etc. [...] schaffen « (Foucault 2004a: 53), wobei sie sich dieses Komplexes und dieser Aufgabe im Sinne eines Familienoberhauptes bzw. eines Schiffsführers unter Regulierungs-, Steuerungs- und Managementgesichtspunkten annimmt (Foucault 2004a: 144-146, 488). Gouvernementalität, so kann nunmehr geschlossen werden, umfasst und bezeichnet das Politische ${ }^{27}$ insgesamt - unter den Bedingungen seiner exekutiven Einfärbung und Inklination. Die exekutive Logik erfährt hierdurch wiederum eine gegenständliche wie programmatische Erweiterung im Sinne einer weitreichenden bevölkerungspolitischen Rationalität.

Dass die Gouvernementalität vornehmlich eine umfassende Rationalität des Regierens, gleichsam eine den gesamten sozialen Bereich - inklusive der Konstituierung ihrer Subjekte - umfassende politische Vernunft ist, die weder in der organisierten exekutiven Staatsgewalt ihren Ursprung hat, noch sich auf diese beschränken oder reduzieren lässt, ist ein Unterschied von großer Tragweite, der eine Gleichsetzung von gouvernementaler Macht und exekutiver Staatsgewalt verbietet. ${ }^{28}$ Doch wenngleich dieser Unterschied eine Identifizierung der gouvernementalen Macht mit der durchführenden Gewalt verwehrt, steht er der Feststellung einer besonderen Nähe der Gouvernementalität zur Exekutive und mithin der dargelegten staatstheoretischen Anschlussfähigkeit Foucaults in diesem Punkte keinesfalls im Wege. Vielmehr scheint just in der gleichzeitigen theoretischen Berücksichtigung der besonderen Nähe der gouvernementalen Machtkonzeption zur staatstheoretischen Logik der Exekutive einerseits sowie des über die institutionelle und rechtsförmige Begrenzung der Regierung hinausgehenden Wirkungsfelds der Gouvernementalität andererseits nicht nur eine theoretisch fruchtbare Verbindung zwischen Gouvernementalität und Regieren (i.e.S.), sondern auch der analytische Mehrwert für die empirische Betrachtung grenzüberschreitender Beziehungen zu liegen.

\section{Die Entgrenzung der Regierung in der internationalen Politik}

Wenn nun gezeigt werden konnte, dass das Foucaultsche Konzept der gouvernementalen Macht aufgrund ihrer exekutiven Einfärbung und Ausrichtung an den staatstheoretischen Begriff der Regierung anschlussfähig ist, bzw. als mit diesem verbunden gelten muss, dann ist dies zum einen ein theoretisches Ergebnis, das dazu beitragen kann, die sowohl bei Foucault selbst als auch in der Gouvernementalitätsfor-

27 Zur Unterscheidung zwischen der Politik und dem Politischen, die nicht zuletzt auf ein Unbehagen zurückgeht und sich skeptisch zeigt, dass das, was heute als Politik bezeichnet wird, schon tatsächlich alles Politische umfasst, vgl. Mouffe (2005: Kap. II, III) sowie für die aktuelle Diskussion zur sogenannten politischen Differenz insgesamt Bedorf/Röttgers (2010).

28 Es mag - neben dem für Foucaults Arbeitsweise durchaus typischen Willen zur grundsätzlichen Abgrenzung von tradierten Modellen und Konzepten - dieser wesentliche Unterschied sein, der dazu geführt hat, dass sowohl Foucault als auch die auf ihn zurückgehenden governmentality studies über lange Zeit den Staat insgesamt sowie speziell die Funktion und Bedeutung institutioneller und rechtsförmiger Machtphänomene kaum bzw. lediglich in dichotom-abgrenzender Absicht berücksichtigt haben. Vgl. Fußnote 10. 
schung festzustellende Unterberücksichtigung des Staates und seiner Institutionen in einem konzeptionell schärferen Sinne, d.h. jenseits allgemeiner Referenzen zu Sammelbegriffen wie Souveränität oder souveräner Macht, aufzubrechen. Die Feststellung, dass die Gouvernementalität einerseits auf die exekutive Staatsgewalt bezogen ist, andererseits aber als eine umfassende politische Rationalität sowohl gegenständlich, räumlich als auch institutionell über diese hinausgeht und diese gleichsam entgrenzt und zugleich konkretisiert, scheint mir zum zweiten aber in empirischer Absicht die besondere Eignung einer solchen Perspektive für die Analyse und Kritik entgrenzten Regierungshandelns in der internationalen Politik nahezulegen.

Dass grenzüberschreitende Politik auch in globalisierten Zusammenhängen nicht nur weiterhin stark von Staaten geprägt wird (vgl. Müller 2009: 235-237), sondern spezieller - noch immer ein Aktivitätsbereich vor allem von staatlichen Regierungen ist (Held 2007: 124), ist eine Feststellung, die schon vor dem vermeintlichen Comeback des Staates in der Finanzmarkt- und Wirtschaftskrise seit 2008 Gültigkeit hatte. So hat Ernst-Otto Czempiel (1999: 35) festgestellt und es mit Blick auf die binnenstaatliche Gewaltenteilungspraxis in zeitgenössischen Demokratien als demokratisch defizitär kritisiert, dass selbst unter Bedingungen der verstärkten Inter-, Trans- oder gar Supranationalisierung politischer Aktivitäts- und Entscheidungsmuster die grenzüberschreitende Politik weiterhin von einer starken Regierungslastigkeit gekennzeichnet sei. Dieser Befund tritt ebenfalls in der Kritik Robert O. Keohanes (2006: 3, 10-11) an der mangelnden demokratischen Legitimität eines regierungszentrierten Multilateralismus' sowie schließlich in sicherheitspolitischen Studien (vgl. Schuck 2011; Calder/Fukuyama 2008) empirisch zutage. Darüber hinaus hat Klaus-Dieter Wolf (1999: 333-363) gezeigt, dass wir es nicht lediglich mit einem fortgesetzten und anachronistischen Primat der Exekutive trotz Globalisierung zu tun haben, sondern die Strukturen und Dynamiken grenzüberschreitender Politik vielmehr selbst eine Stärkung nationaler Regierungen favorisieren. Dass Machtverschiebungen von legislativen zu exekutiven Organen gerade aufgrund der Gleichzeitigkeit einer Intensivierung grenzüberschreitender Beziehungen bei fortgesetztem exekutivem Vorrang in diesem Bereich stattfinden, habe ich an anderer Stelle aus einer staatstheoretischfunktionalen Perspektive diskutiert (Vasilache 2011b). Schließlich hat - allerdings in affirmativer Absicht und ganz im Sinne traditioneller Staatsrechtslehre (Jellinek 1966: 617) - das Bundesverfassungsgericht (BVerfG) festgeschrieben, dass die $\gg$ Regierung im Bereich auswärtiger Politik einen weit bemessenen Spielraum zu eigenverantwortlicher Aufgabenwahrnehmung« (BVerfG 2001: Abs. 149) habe.

Nun scheint mir ein analytischer Mehrwert der Verbindung einer klassisch-gewaltenteilungsbezogenen Sicht mit einer gouvernementalitätstheoretischen Perspektive darin zu bestehen, sowohl die institutionelle Stärkung nationaler Regierungsapparate als auch die simultane Ausdehnung exekutiver Rationalitäten und Logiken über die institutionalisierte exekutive Gewalt hinaus erfassen und beschreiben zu können. Denn während die soeben angeführten Kritiken des fortgesetzten Primats der Regierung in der grenzüberschreitenden Politik aus einer institutionellen Perspektive argumentieren, erlaubt eine gouvernementalitätstheoretisch inspirierte Sicht nicht lediglich die Untersuchung der Machtverschiebung von legislativen zu exekutiven Or- 
ganen, sondern darüber hinaus auch die Betrachtung der Kolonisierung legislativer und judikativer Organe sowie (zivil-)gesellschaftlicher privater und semi-privater Akteure und der entsprechenden Diskurse durch eine ordnungspolitisch fokussierte exekutive Rationalität und Maßnahmenlogik.

So entspricht die thematische, strukturelle und institutionelle Offenheit und Ungebundenheit der gouvernementalen Macht, die ja als umfassende Rationalität nicht zuletzt diskursiv funktioniert, der kommunikativen Entgrenzung und Öffnung (beinahe) aller Politikfelder für ihre inter- und transnationalen Implikationen (vgl. Müller 2002: 7). Diese Bewegungsfreiheit der Gouvernementalität korrespondiert insgesamt mit dem »zunehmenden Umfang und [...] [der] Intensivierung von Verkehrs-, Kommunikations- und Austauschbeziehungen über nationale Grenzen hinweg« (Habermas 1998: 101), kurzum: mit den Strukturen und Dynamiken der Politik in der Globalisierung. ${ }^{29}$ Geht man ins systematische Detail, so lassen sich sechs wesentliche Kongruenzen zwischen der exekutiv eingefärbten Gouvernementalität und den Strukturen globalisierter Politik feststellen, die einer verwaltungsförmigen »Gouvernementalisierung des Staates« (Foucault 2004a: 163, Hervorh. dort) und der Politik insbesondere in den grenzüberschreitenden Beziehungen dienlich zu sein versprechen:

- So werden Gouvernementalität und Globalisierung erstens durch ihre thematische Offenheit und damit durch ihre Politikfeldungebundenheit geeint. Beide sind nicht auf bestimmte Politikfelder oder -bereiche beschränkt.

- Zweitens handelt es sich sowohl bei der gouvernementalen Ratio der Politik als auch bei globalisierten Strukturen im weiteren Sinne um entgrenzte Phänomene sowie um Phänomene der Entgrenzung, da sie nationalstaatliche Territorialgrenzen in inhaltlicher und kommunikativer Hinsicht transzendieren bzw. die Grenzüberschreitung eines ihrer wesentlichen Charakteristika ist.

- Gouvernementalität und Globalisierung korrespondieren drittens in ihrer institutionellen Ungebundenheit. Während für die gouvernementale Macht die formale Institution lediglich eine ihrer speziellen Ausdrucksformen ist, zeichnet sich globalisierte Politik durch ein Mit- und Gegeneinander einer Vielzahl von formellen und informellen Akteuren sowie Akteurstypen (öffentlichen, semi-privaten, privaten) aus, wobei die institutionellen Strukturen und Dynamiken keineswegs festgelegt sind, sondern sich in permanentem Fluss und ständiger Aushandlung befinden. ${ }^{30}$

- Die exekutive Inklination der gouvernementalen Macht korrespondiert - wie ausgewiesen - viertens mit der Regierungslastigkeit globalisierter Politik und

29 Um nicht wiederholt von zunehmender Inter-, Trans- und Supranationalisierung und Regionalisierung zu sprechen, wird sich, wenn im Folgenden von Globalisierung die Rede ist, dieser Begriff auf die soeben zitierte, weite Definition von Jürgen Habermas beziehen - mit der mein Begriff der grenzüberschreitenden Beziehungen weitgehend kongruent ist -, selbst wenn die genannten Aspekte nicht immer von globaler Bedeutung oder nicht in allen Weltregionen in gleichem Maße zu beobachten sind.

30 I.d.S. betont Philip G. Cerny, dass »governmentalization does not necessarily mean the strengthening or growth of existing institutions of global governance as such « (Cerny 2010: 187, vgl. 186-187). 
ihrem Fokus auf das Partikulare, ihrer konkreten Gegenstandsbezogenheit, ihrer Schwerpunktsetzung im Bereich der Problemerkennung, -behandlung und -lösung, ihrem technischen Implementierungsfokus sowie ihrer - oben dargelegten - Gesetzesferne.

- Fünftens sind Gouvernementalität wie Globalisierung gleichermaßen als Bewegungen der Beschleunigung zu begreifen. Während Globalisierung nicht zuletzt in einem Anstieg der Anzahl und der zeitlichen Dichte von grenzüberschreitenden Beziehungen besteht (Rosa 2005: Teil 4), geht die gouvernementale Macht als eine auf Effizienz ausgerichtete Ökonomie der Politik mit einer Beschleunigung und zeitlichen Verdichtung einher.

- Schließlich korrespondiert sechstens die gouvernementale Sicherheitsfürsorge mit der traditionell hohen Bedeutung der Sicherheit in der Außenpolitik sowie in analytischer Hinsicht - ebenfalls in der Disziplin der IB.

Es gilt zu betonen, dass diese systematischen Korrespondenzen, die die Strukturen globalisierter Politik für die Ausdehnung exekutiv-gouvernementaler Logiken prädestinieren, keine feste Institutionalisierung implizieren und keineswegs zwingend oder alleine in formellen Kompetenzerweiterungen bestimmter Staatsorgane zulasten anderer Staatsorgane aufgehen. Wollte man die genannten Kohärenzen zwischen Gouvernementalität und Globalisierung auf einen Punkt bringen, so besteht die wesentliche Gemeinsamkeit in einer exekutiv vorgefärbten, grenzüberschreitenden Ungebundenheit. Wenn also globalisierte Strukturen einer Ausdehnung exekutivlastiger Politik zuträglich zu sein scheinen, dann ist damit nicht immer und sicherlich nicht alleine eine formale oder juridische Macht- und/oder Kompetenzerweiterung von exekutiven Organen zulasten legislativer und/oder judikativer Organe gemeint. Daraus, dass die gouvernementale Macht eine »Regierungsform [ist], die sich des gesamten Staates bemächtigt«(Foucault 2004a: 142), lässt sich vielmehr schließen, dass das systematische Potenzial der gouvernementalen Macht aufgrund ihrer exekutiven Einfärbung und ihrer gleichzeitigen institutionellen Ungebundenheit in einem verwaltungslogischen mainstreaming der Politik besteht.

Allerdings bedeutet dies zugleich, dass gouvernementale Macht und politische Institutionen keinesfalls in einem Ausschlussverhältnis zueinander stehen, sondern sich gouvernementale Macht institutionell realisieren lässt - aber eben nicht auf Institutionen beschränkt ist. In der Tat wäre es, wie sinngemäß auch Jonathan Joseph (2010: 241, 243) betont, ein Fehler, aus der institutionellen Ungebundenheit der Gouvernementalität ihre grundsätzliche Inkompatibilität mit Institutionen zu schließen - wie dies in einer subjektologischen bzw. biopolitischen Verkürzung des Gouvernementalitätsfokus insinuiert wird. Vielmehr besteht die Ungebundenheit der Gouvernementalität darin, sich von der Regierung (im begrifflich engeren Sinne), ihren traditionellen Institutionen und Instrumenten sowie ihrer nationalen Geltungsbeschränkung ablösen und zugleich »die Apparatur des Staates « (Lemke 2007: 54) nutzen und affizieren zu können, insbesondere wenn es um zwangsbewährte Durchsetzung geht. Als allgemeine Ratio der Politik vermag sich die Gouvernementalität potentiell auf das gesamte institutionelle Setting einer polity sowie auch den privaten Raum zu 
erstrecken und diese zu überstrahlen. Insgesamt bezieht sich Gouvernementalität, um es in klassischen Begriffen auszudrücken, gleichermaßen auf polities, policies und politics. Diese institutionelle und thematische Offenheit der Gouvernementalität kann einer Expansion exekutiver Logiken insbesondere durch die Strukturen sowie im Rahmen globalisierter Politik als förderlich angesehen werden. Daher erlaubt eine exekutiv-gouvernementalitätstheoretische Perspektive m.E. die analytische Erfassung und in einem Rortyschen Sinne »Neubeschreibung« (Rorty 1992: 128, vgl. 128-130, 153-155) von Phänomenen einer verwaltungslogischen Rationalisierung in der grenzüberschreitenden Politik.

\section{Exekutive Gouvernementalität und grenzüberschreitende Sicherheit}

Nachdem zunächst die besondere Nähe der Gouvernementalität zur staatstheoretischen Systematik der Exekutive und anschließend die Korrespondenz einer solchen integrativen Perspektive mit den Grundstrukturen und -dynamiken grenzüberschreitender Politik ausgewiesen werden konnten, soll der Blick sich nun explorativ auf die mögliche empirische Anwendung dieses analytischen Rahmens richten. Wie eingangs betont, konzentrieren sich die bisherigen Adaptionen des Gouvernementalitätsansatzes im Sachbereich der internationalen Politik oft im Sinne einer grand theory auf den Ausweis einer allgemeinen »global governmentality« (Lipschutz/Rowe 2005: $15)^{31}$ und eines umfassenden gouvernementalen Wandels, in der die »raison d'État is increasingly giving way to raison du monde« (Cerny 2010: 157, Hervorh. dort, vgl. 5, 157-159, 173-192) - oder sie verbleiben innerhalb eines engeren gouvernementalitätstheoretischen Paradigmas und gehen über einen subjekttheoretischen und biopolitischen Fokus kaum hinaus. ${ }^{32}$ In Untersuchungen, die sich detaillierter auf die Gouvernementalitätstheorie beziehen, wird in gegenständlicher Hinsicht meist auf die neoliberale Ökonomisierung globaler Politik fokussiert, ${ }^{33}$ wie insgesamt die governmentality studies (insbesondere zu Beginn) einen deutlichen Schwerpunkt in der Erforschung des ökonomischen Liberalismus aufweisen (vgl. Bröckling et al. 2000; Opitz 2004). Eine recht allgemeine Bezugnahme zur Foucaultschen Theorie ist schließlich in den critical security studies (CSS) festzustellen. Zwar weisen die CSS einerseits einen gegenständlichen Fokus auf Sicherheitspolitiken auf, andererseits erschöpfen sich Rekurse auf Foucault hier meist in allgemeinen affirmativen Verweisen

31 Kritisch äußert sich diesbezüglich aktuell Joseph (2011), der die sozialen Voraussetzungen als nicht gegeben ansieht, um von globaler Gouvernementalität von Bevölkerungen zu sprechen.

32 Vgl. z.B. die Beiträge in Dillon/Neal (2008), in de Larrinaga/Doucet (2010a) sowie Bigo/ Tsoukala (2008) und Bigo (2008).

33 Vgl. Lipschutz/Rowe (2005: 58-60); Cerny (2010: 157-174); Fougner (2008); TraubWerner (2007). 
auf die Bedeutung von Diskursen und ihre soziale Konstruiertheit. ${ }^{34}$ Zugleich beziehen sich die CSS schwerpunktmäßig aufFoucaults frühere Diskurs- und Machttheorie und nehmen einen vornehmlich auf das Individuum fokussierten Blick ein (vgl. Linklater 2005: 122) - was nun aber just aus einer exekutiv-gouvernementalen Perspektive kritisiert werden kann.

Gemein ist diesen Rekursen auf Foucaultsche Ansätze, dass sie Foucault in seiner expliziten Abwendung von staatstheoretischen Begriffen folgen und die exekutive Inklination des Gouvernementalitätsansatzes nicht in ihrer systematischen und konzeptionellen Bedeutung berücksichtigen. Im Unterschied zu den Absichten einer umfassenden Großtheorie sowie in Ergänzung politikökonomischer und überwiegend subjektfokussierter Forschungsschwerpunkte soll aus der bisher dargelegten Verbindung von Gouvernementalität und exekutiver Logik daher nun der Blick auf einige zunächst als unzusammenhängend erscheinende Phänomene aus dem Bereich der grenzüberschreitenden Sicherheit gerichtet werden. Die Würdigung dieser Beispiele aus einer solchen Perspektive kann nicht nur wesentliche strukturelle Parallelen zwischen ihnen offenlegen, sondern die Funktionsweise exekutiv-gouvernementaler Sicherheitspolitik als einer reziproken Affizierung und Verschmelzung gouvernementaler und exekutiver Rationalitäten einsichtig machen. Dabei verspricht eine auf exekutive Gouvernementalität konzentrierte Optik die Erfassung biopolitischer Transformationen der Sicherheitspolitik, ohne aber die Persistenz traditioneller, institutioneller, staats- und militärfokussierter Sicherheitspolitiken unterschätzen oder ignorieren zu müssen.

Eine exekutive Gouvernementalisierung politischer Inhalte und Dynamiken ist insbesondere in sicherheitspolitisch relevanten Politikfeldern festzustellen, und zwar in zweifacher Hinsicht. Zum einen entfaltet sie sich im Feld der Sicherheitspolitik. Sie kann hier ihre ordnungspolitische und auf das Partikulare orientierte Maßnahmenund Durchführungslogik besonders gut zur Geltung bringen, da der Bereich der (inneren wie grenzüberschreitenden) Sicherheitspolitik schon staatstheoretisch (Anter 2003; Vasilache 2007) und in der politischen Praxis ein Politikfeld bildet, das von einer politischen Prärogative der Exekutive gekennzeichnet ist. Zum zweiten besteht die administrative Gouvernementalität zugleich auch darin, den Gegenstandsbereich der Sicherheit auszudehnen und neue, ehedem nicht der Sicherheitspolitik zugehörige Fragen, Probleme und ggf. ganze Politikfelder unter das Dispositiv der Sicherheit zu stellen. Dass Sicherheit sowie Sicherheitspolitik keine apriorisch feststehenden Bereiche und Akteure umfassen, sondern vielmehr Produkte einer diskursiven securitization darstellen, ist überzeugend von Barry Buzan et al. (1998: 23-25, 195) darge-

34 Vgl. die Beiträge in Booth (2005) sowie i.d.S. auch Buzan/Hansen (2009: 205-206). Vgl. für eine Kritik an der einseitigen Überbetonung sozialer Konstruiertheit von Sicherheitsdiskursen in den frühen CSS Buzan et al. (1998: 34-35) sowie für eine Kritik daran, dass die CSS bisweilen nichtintentional in ein traditionelles und objektivistisches Sicherheitsverständnis zurückfallen, ebenfalls Buzan et al. (1998: 204). Für die später stärkere Abgrenzung der CSS vom Aspekt der sozialen Konstruiertheit vgl. Buzan/Hansen (2009: 207). Zugleich ist der Kopenhagener Schule von der Pariser Schule ein noch zu objektivistisches Verständnis von Sicherheit und Versicherheitlichung vorgeworfen worden (Bigo/Tsoukala 2008: 5). 
legt worden. An ihren Ausweis der diskursiven Expansionsmöglichkeit des Sicherheitsparadigmas ist eine exekutiv-gouvernementale Perspektive zwar durchaus anschlussfähig. Die exekutiv-gouvernementale Ratio funktioniert in der Tat durch und basiert auf diskursiver Versicherheitlichung. ${ }^{35}$ Sie geht aber zugleich über diese hinaus, indem sie erstens nicht in jedem Fall genuine Sicherheitsdiskurse produziert, sondern auch unterhalb der Schwelle einer unmittelbaren securitization ganzer Politikfelder einzelne Fragen, Probleme und Diskurse affiziert. Schließlich geht exekutive Gouvernementalität zweitens über Diskurse überhaupt hinaus, indem sie sich gleichzeitig auf Diskurse, Institutionen und Akteure bezieht. Ihre Versicherheitlichungsleistung erschöpft sich nicht in einer diskursiven Ausdehnung des Sicherheitsbereichs, sondern besteht in der Entfaltung ihrer eigenen, besonderen politischen Vernunft.

Dies bedeutet letztlich auch, dass Sicherheitspolitik nicht genuin, sondern bestenfalls taktisch als eigenes Politikfeld umrissen wird. Die exekutiv-gouvernementale Sicherheitspolitik unterliegt daher keiner engen, (neo-)realistischen Begrenzung auf Verteidigungs- und Militärpolitik, sondern kann potenziell in jedem Politikfeld als »Sicherheitsdispositiv« (Foucault 2004a: 19) zur Entfaltung kommen. Im Rahmen ihrer Sicherheitsdispositive zielt die administrative Maßnahmenrationalität der Gouvernementalität letztlich auf die Herstellung und das Gedeihen einer guten Ordnung überhaupt ab. In dieser Ordnung haben alle Menschen und Objekte ihren Platz und sollen - gleichsam in harmonischer Effektivität - »zu einem für jedes dieser zu regierenden Dinge angemessenen Ziel« (Foucault 2004a: 149, vgl. 148-150) gelenkt werden. Wenn der Sicherheit schon aus klassischer staatstheoretischer Perspektive eine hervorgehobene Bedeutung zugekommen ist - und im Kontraktualismus als Ziel und raison d'être die Staatskonstituierung rechtfertigt -, dann ergänzt und verdeutlicht der gouvernementale Blick, dass Sicherheit nicht nur Gegenstand und Ziel, sondern auch »technisches Instrument« (Foucault 2004a: 162) moderner Regierungsrationalität ist, durch das gesellschaftliche Milieus angeleitet und gesteuert werden (Foucault 2004a: 39-44, 53).

Dabei ist das exekutiv-gouvernementale mainstreaming sicherheitspolitischer Inhalte, Strukturen und Prozesse wesensmäßig grenzüberschreitend, und zwar in mehrerlei Hinsicht, da es weder gegenständlich oder territorial, noch institutionell oder organisatorisch festgelegt ist. Es ist potentiell auf jede politische Frage anwendbar, in seiner Aktivitäts- und Interventionslogik nicht nationalstaatlich begrenzt und nicht an bestimmte Staatsinstitutionen gebunden, wobei auch die klassische staatstheoretische Unterscheidung zwischen dem privaten und dem öffentlichen Raum suspendiert wird. Schließlich ist das exekutiv-gouvernementale mainstreaming in jenem Sinne teleologisch, dass es der Erreichung des Ziels der Sicherheit einen Vorrang einräumt gegenüber den verwaltungstechnischen Instrumenten der Zielerreichung. Da »this way of thinking is [...] obsessed with efficiency« (Castel 1991: 295), finden sich die

35 Für eine Darlegung, wie Sicherheitsdiskurse aus einer gouvernementalitätstheoretischen Perspektive in ihrer Binnenstruktur funktionieren, vgl. Dillon (2004: 84) sowie umfassend Dillon/Neal (2008) und Dillon/Reid (2009). 
Instrumente der Sicherheitspolitik aber ironischerweise just durch ihre teleologische Unterordnung in ihrer Relevanz aufgewertet. Auf die Frage des Rechts bezogen bedeutet dieser letzte Punkt, dass dem Gesetz kein normativer Vorrang vor anderen Instrumenten des Regierens eingeräumt wird - seien es (Durchführungs-)Verordnungen und Anordnungen, Ratschläge, Benchmarks, kulturelle und gewohnheitsmäBige Normen (und ihr Wandel), etc. - und damit institutionalisierte Gewaltenteilung zugunsten einer exekutiven Inklination politischen Handelns als Management insgesamt unterwandert wird. Man könnte in Anlehnung an die von Barry Buzan, Ole Wæver und Jaap de Wilde (1998: 195) hervorgehobene Unterscheidung von »security issues from merely political ones« sagen, dass in Phänomenen der exekutiv-gouvernementalen securitization eben diese Unterscheidung aufgehoben wird und Politik in einer umfassenden verwaltungsförmigen Sicherheitsgewährleistung aufgeht. Dies setzt allerdings zugleich voraus, dass - gegen den (in-)securitization-Ansatz der Pariser Schule (Bigo/Tsoukala 2008: 5; Bigo 2008: 113; C.A.S.E. Collective 2006: 255) - an dieser Unterscheidung, die durch eine exekutiv-gouvernementale Versicherheitlichung suspendiert wird, konzeptuell festgehalten wird.

Die in den vergangenen Jahrzehnten - insbesondere aber seit dem Ende des OstWest-Konfliktes - nicht zuletzt im europäischen Raum zu beobachtende Expansion der Sicherheitspolitik bzw. sicherheitspolitische Neubeschreibung von politischen Fragen und ganzen Politikfeldern, die ehedem nicht als Teil der Sicherheitspolitik angesehen worden sind, scheint nun aus einer auf exekutive Gouvernementalisierung fokussierten Perspektive gut beschreibbar zu sein. Denn so ist in den vergangenen Jahren nicht nur offensichtlich geworden, dass das inklusive Potenzial eines erweiterten Sicherheitsbegriffs ${ }^{36}$ nahezu unbegrenzt ist - so dass es, um lediglich einige illustrierende Beispiele zu geben, »Food Security« (FAO 1996), »Energy Security« (Moran/Russell 2009), die Verbindungen von »Environmental, Human, Energy, Food, Health and Water Security Concepts« (Brauch et al. 2009), »Bio-Security« (Jones 2006) oder das Ziel einer »safer future« durch »Global public health security« (WHO 2007) und schließlich »Cultural Security« (Friedman/Randeria 2004) einzubeziehen vermag. Vielmehr sind die meisten dieser sicherheitspolitischen Expansionen auch nicht mehr alleine auf Staaten gerichtet, sondern ebenso auf Bevölkerungen und ihre Individuen, die einmal als Sicherheitsobjekte, ein andermal als -subjekte oder als beides zugleich adressiert werden. Ein eingängiges Beispiel bildet hier das humansecurity-Konzept, das sich in Wissenschaft und politischer Praxis einer weiten Verbreitung und Anhängerschaft erfreut und die Vor- und Nachteile des »job-sharing « (UNDP 1994: 39), den Konsum von »narcotic drugs and a growing sense of individual isolation« (UNDP 1994: 2) sowie schließlich gar Verkehrsunfälle und individuelle Kriminalität innerhalb von stabilen Staaten mit funktionierendem Strafrechtssystem unter dem vagen und weiten Schlagwort des »human distress« (UNDP 1994: 30) als

36 Für die Diskussion traditioneller und erweiterter Begriffe der Sicherheit vgl. noch immer Buzan et al. (1998: 2-20, 207-209) sowie Buzan/Hansen (2009). 
Gegenstände internationaler Sicherheitspolitik definiert. ${ }^{37}$ Die exekutive Gouvernementalität kann sich in einem solchen Kontext auf Bevölkerungen in ihrer statistischen Gesamtheit richten, deren Individuen als Hauptadressaten gouvernementaler Sicherheitsfürsorge und -maßnahmen fungieren und durch eine mathematisch-technische Logik der Risikoberechnung und vielfältige Normalisierungsstrategien als regierbare Sicherheitssubjekte und -objekte konstituiert werden (vgl. Demirović 2008: 236-244; Dean 1999: 177). Dieser normalisierende Fokus wird aber zugleich von einer Expansion des materialen Regierungsapparats begleitet, wobei im Falle individueller Renitenz gegen derlei fürsorgende Regulierung das institutionell-repressive Arsenal der Exekutive aktiviert werden kann.

Die Kontrolle von »)suspect< products « (Castel 1991: 289) kann als eingängiges Beispiel einer Sicherheitslogik gelten, die in der gefahrenabwehrenden Fürsorge gegenüber Bevölkerungen besteht, sich sowohl als umfassende gesellschaftliche Rationalität als auch institutionell in Gesetzen, Einzelmaßnahmen, Verordnungen etc. realisiert und auf die Normalisierung der Individuen abzielt - während sie Gegenstand traditioneller internationaler Politik geworden ist: Nicht nur die besondere Schwerpunktsetzung der schwedischen Ratspräsidentschaft in der EU auf das Thema des Alkoholkonsums (vgl. Council of the European Union 2009), sondern auch die Tatsache, dass die Weltgesundheitsorganisation (WHO) im Kampf gegen den globalen Tabakkonsum eine ihrer wesentlichen Aufgaben sieht und seit 2008 nun auch keine Tabakkonsumentinnen und Tabakkonsumenten mehr einstellt (WHO 2008: 1-2), ${ }^{38}$ ist in den hier dargelegten theoretischen Begriffen zu fassen. Hervorzuheben ist die letztgenannte Regelung auch aufgrund ihrer formalen Einbettung in einen rechtlich prekären zwischenstaatlichen Raum. Denn so ist diese Einstellungspolitik zwar einerseits institutionell und juridisch fixiert und im Rahmen der WHO geltende Norm, während andererseits und zugleich ein solcher Ausschluss innerhalb zahlreicher Mitgliedsstaaten der WHO, die diese Organisation überhaupt erst durch ihre im nationalen Recht kodifizierte Mitgliedschaft völkerrechtlich konstituieren und zum Handeln ermächtigen, zweifellos rechtswidrig ist. ${ }^{39}$

An diesen Beispielen wird indes nicht nur die territoriale und auch rechtsgeltungsmäßige Ungebundenheit der exekutiv-gouvernementalen Sicherheitspolitik deutlich. Es zeigt sich darüber hinaus, dass diese Ungebundenheit bisweilen einer der Exekutive institutionell förderlichen Außenpolitisierung von Problemen und ganzen Politikfeldern Vorschub leistet, die durchaus innerhalb des Rahmens demokratischer

37 Vgl. UNDP (1994: 30-31) sowie für eine detaillierte Diskussion des human-security-Konzepts aktuell Schuck (2011) sowie mit Blick auf Europa Vasilache (2011a) und hinsichtlich seiner biopolitischen Implikationen de Larrinaga/Doucet (2008).

38 Wohlgemerkt geht es bei dieser Maßnahme ausdrücklich nicht um das Rauchen am Arbeitsplatz, sondern um den möglichen Tabakkonsum der Mitarbeiterinnen und Mitarbeiter überhaupt, d.h. in der Freizeit und in privaten Kontexten (WHO 2008: 1).

39 Wir haben es in diesem Punkte allerdings nicht allein schon deshalb mit supranationaler Macht zu tun, weil die WHO einen Vorrang vor dem nationalen Recht ihrer Mitgliedsstaaten, d.h. ihrer konstitutiven Einheiten, geltend macht. Denn so basiert eine solche Maßnahme zwingend darauf, dass sie von den Staaten geduldet und der WHO eine solche Regelung zugestanden wird. 
Rechtsstaaten angegangen werden können. Es tritt außerdem noch ein weiterer Punkt hervor, der für die exekutive Gouvernementalisierung charakteristisch und ihrer Entfaltung gerade in der Sicherheitspolitik besonders förderlich ist. So wird die verwaltungslogische Überlast der Gouvernementalität im Sachbereich der Sicherheit auch durch ihre präventive Ausrichtung sowie den hiermit zusammenhängenden Imperativ einer umfassenden staatlichen, gesellschaftlichen und individuellen »preparedness" (Lentzos/Rose 2008: 83, Hervorh. dort) deutlich. Denn während das Gesetz vornehmlich in allgemeinen Verboten besteht und im Falle der Übertretung im Nachhinein sanktionierend in Erscheinung tritt, muss das Ziel der Sicherheit idealerweise in der präventiven Abwendung, Risikominimierung sowie der Vorausschau und Wirkungsminimierung von drohenden Gefahren bestehen. Dies ist der Fall im Bereich (klassischer) militärischer Sicherheitspolitik, wenn (vermeintliche, mögliche oder reale) Bedrohungen schon vor Eintritt beantwortet werden oder im Bereich volksgesundheitlicher Risiken, die - wie beispielsweise in Frankreich - durch verpflichtende Warnhinweise auf allen Lebensmittelreklamen minimiert werden sollen. ${ }^{40}$ In diesem Zusammenhang ist der Begriff des Risikos ein konzeptioneller Schlüsselbegriff: »Risk is a way [...] of ordering reality, of rendering it into a calculable form « (Dean 1999: 177). ${ }^{41}$ Dabei ist dem exekutiv-gouvernementalen Konzept des Risikos die präventive Ausrichtung als naheliegender Realisierungsmodus bereits inhärent (Foucault 2004b: 101; vgl. Vasilache 2011a) und daher nicht als neue, ${ }^{42}$ sondern als aktuell dominante Form des Risikomanagements anzusehen. Insbesondere im Imperativ der Prävention und Risikominimierung schlägt sich der rationalistisch-technische Zugriff der exekutiv-gouvernementalen Macht nieder, die sicherheitspolitische Diskurse und Politiken mit bevölkerungspolitischen Kosten-Nutzen-Kalkülen, Grenzkostenberechnungen, statistischen Prognosen etc. reguliert, managt und durchzieht, während sie sich zugleich in einem hohen Maße institutionell in gesetzten Vorgaben, Anweisungen und Verboten, gleichsam behördlich und oft repressiv realisiert.

Einige der soeben gegebenen Beispiele mögen als Marginalien betrachtet werden. Nichtsdestoweniger werden in ihnen alle genannten charakteristischen Merkmale einer exekutivlogischen Gouvernementalität sichtbar - inklusive ihrer internationalen Entgrenzung, die mit Blick auf Lebensstilfragen sich ja keinesfalls von selbst versteht. Gleichwohl bezieht sich die exekutiv-gouvernementale Logik in der Sicherheitspolitik auch auf Gegenstände, die schon in einer klassischen, gleichsam engen Fassung des Sicherheitsbegriffs als sicherheitsrelevant gelten. Dass sich exekutive gouvernementale Rationalitäten keineswegs auf vermeintlich weiche Sicherheitsfragen alleine beziehen, verdeutlicht Yves Winter, der in seiner Diskussion des Zusammenspiels von Privatisierung (Winter 2008: 59-61), Individualisierung (63-65) und aktueller »Risiko- und Sicherheitslogik« (66, vgl. 66-67) Hinweise auf die »Gouvernementalität der `neuen Kriege« (49) liefert. In diesem Sinne betont auch Vivienne Jabri, dass

40 Zur Vermeidung von Missverständnissen: Durch den Ausweis dieser strukturellen Kongruenz ist keineswegs eine generelle Gleichsetzung der beiden genannten Beispiele behauptet.

41 Für den Risikobegriff vgl. Aradau (2004: 264-269).

42 Anderer Ansicht sind Aradau/Van Munster (2007: 91, 102-106). 
Foucaults »conception of liberal governmentality is informed by an analytic of war" (2006: 56). ${ }^{43} \mathrm{Zu}$ ergänzen ist in beiden Aussagen die exekutive Erdung der Gouvernementalität, die sich beispielsweise in der gouvernementalen Kulturalisierung bzw. kulturellen Aufladung sowohl von neuen Kriegen als auch von internationalen Territorialkonflikten bei gleichzeitigem Fortbestand klassischer geopolitischer und -strategischer Rationalitäten zeigt.

Dass eine »Kultur der Gefahr« (Foucault 2004b: 102), in der »die Individuen [...] darauf konditioniert werden, ihre Situation, ihr Leben, ihre Gegenwart, ihre Zukunft usw. als Träger von Gefahren zu empfinden« (Foucault 2004b: 101), sich in der Tat nicht lediglich auf vermeintlich weiche Gefahren, sondern ebenso und ganz unmittelbar auf hard issues bezieht und eine exekutive Einfärbung erfährt, wird innerhalb westlicher Staaten in der Bekämpfung grenzüberschreitender Bedrohungen wie des inter- und transnationalen Terrorismus oder der organisierten transnationalen Kriminalität besonders deutlich (Garland 2002; Dillon 2007; Heng/McDonagh 2008). Wenn beispielsweise am Flughafen Houston Reisende mit einer Lautsprecherwarnung überrascht werden, der zufolge »inappropriate remarks or jokes concerning security may result in your arrest «, wird das Individuum zum einen als Gefahren- und damit Repressionsobjekt, gleichsam als »potentially disruptive passenger« (Commission of the European Communities 2005: 6, 15, 17), konstituiert. Zum anderen aber wird es als eigenverantwortliches, aktives und kooperatives Subjekt an der Prävention grenzüberschreitender Gefahren beteiligt. Es handelt sich um eine Maßnahme im Feld der hard security issues, in der eine liberale Normalisierungs- mit einer klassisch-exekutiven Unterbindungsstrategie verschmilzt. Eine Schlüsselforderung an die Individuen scheint dabei die der Kooperativität zu sein. Die Subjekte sollen im Rahmen ihrer liberalen Eigenverantwortlichkeit an der Sicherheitspolitik aktiv kooperieren bzw. sich kooperativ verhalten. Neben das gefährdende Individuum tritt somit nicht nur das gefährdete Individuum, das aktiv an der Gefahrenabwehr partizipiert, sondern auch das unkooperative Individuum, das gleichsam eine Mittelstellung zwischen den vorgenannten Kategorien einnimmt. Das unkooperative Subjekt ist allerdings gerade deshalb ein Fall für den normalisierend-repressiven Exekutivapparat, weil es noch nicht gesetzeswidrig gehandelt hat, sondern lediglich durch fehlende Präventionsbereitschaft auffällig geworden ist. Die durch eine solche Politik generierte Unsicherheit ist dabei Programm. So legte das Heimatschutzministerium der USA bei der Verschärfung der Flugsicherheitsbestimmungen Ende 2009 Wert darauf, dass »die neu erlassenen Maßnahmen [...] >unvorhersehbar « [seien], so dass die Passagiere nicht das gleiche überall erwarten dürfen« (Süddeutsche Zeitung, 26.12.2009). ${ }^{44}$ Erklärtermaßen geht es demnach »um das Regieren von Sicherheit durch Unsicherheit, und zwar genau durch [...] Zweifel, Angst, Unruhe [...], um die für die Sicherung der Sicherheit notwendige Wachsamkeit, Bereitschaft und Vorsorge zu gewährleisten« (Lentzos/Rose 2008: 82, Hervorh. dort; vgl. Daase/Kessler 2007).

43 Jabri (2006: 56-57) hebt schließlich die Notwendigkeit hervor, Foucaults Abwendung von gewaltförmigen Machtphänomenen zurückzunehmen.

44 Bemerkenswert an diesem Zitat ist auch, dass nicht Terroristen, sondern ausdrücklich Passagiere genannt und adressiert werden. 
Bisweilen lässt sich eine exekutive sicherheitspolitische Gouvernementalisierung als mittelbare ausmachen, die in einer Verknüpfung von bestimmten Politikfeldern mit traditionellen Sicherheitsfragen oder -maßnahmen besteht. In diesem Zusammenhang sind beispielsweise die Verhandlungen über das Handelsabkommen über Produktfälschungen Acta zu nennen, in denen die Regierungsvertreter einer Handvoll Staaten ${ }^{45}$ unter dezidierter Abschottung gegen ihre Legislativen ihre ursprünglich handelsrechtliche Zielsetzung mit sachfremden Kompetenzerweiterungen im Sicherheitsbereich zu verbinden suchen (vgl. Frankfurter Rundschau, 14./15.08.2010: 6-7). Solche Verknüpfungen sind beispielsweise auch in der im human-security-Konzept vorhandenen Ununterscheidbarkeit von klassischen und neuen Sicherheitsfragen sowie der Vermengung von internationalen, staatlichen, gesellschaftlichen und individuellen Akteuren und Adressatinnen von Sicherheit angelegt. Und auch wenn - um zwei weitere Beispiele anzuführen - in Europa bildungspolitische Diskurse mit kriminalitätspolitischen Debatten in zunehmendem Maße fusionieren, während kultureller Pluralismus und Einwanderung in Begriffen grenzüberschreitender Sicherheit verhandelt werden, dann zeigt sich darin die Verschmelzung zwischen subjektivierender Normalisierungsmacht, Präventionsprimat, exekutiv-repressiver Logik sowie einer Außenpolitisierung rechtsstaatlich klärbarer Fragen.

Hier wie in den zuvor gegebenen Beispielen tritt auch die öffentlich-private Unentschiedenheit der exekutiv-gouvernementalen Logik hervor. Dass die öffentlichprivate Unentschiedenheit nicht in der Privatisierung öffentlicher Aufgaben (z.B. durch private military companies) aufgeht, sondern zugleich in der Politisierung und Öffentlichwerdung ehedem privater Räume und Gegenstandsbereiche besteht, ist an den Beispielen der sicherheitspolitischen Adressierung der Individuen deutlich geworden und manifestiert sich insbesondere auch in den zahlreichen polizeilichen Überwachungsinstrumenten, die in den letzten zehn Jahren zur Gefahrenabwehr eingeführt worden sind - und von allen erdenklichen Einzelmaßnahmen bis hin zur Institutionalisierung neuer Exekutivorgane wie des Department of Homeland Security in den USA oder der Europäischen Agentur für die operative Zusammenarbeit an den Außengrenzen (Frontex) reichen. Wenn die eingeführten Maßnahmen und Institutionen dabei zum einen oft eine geringe Halbwertszeit haben sowie zum zweiten in ihrer Aufgabenstellung, ihren Kompetenzen und ihrer nationalen, internationalen wie transnationalen Einsatzreichweite bewusst dynamisch bzw. vage gehalten sind (vgl. Vasilache 2011a), dann entspricht dies genau der allgemeinen (gegenständlichen, territorialen, öffentlich-privaten) Unentschiedenheit und Offenheit exekutiv-gouvernementaler Rationalität. Zugleich ist diese Offenheit aber mit einem hohen $\mathrm{Ma} ß$ an Verbindlichkeit und Gewissheit ihrer behördlichen und auch repressiven Handlungsbereitschaft verbunden.

Die in den dargelegten Beispielen offensichtliche Verschmelzung zwischen präventiver Normalisierung, Fürsorge und Repression verdeutlicht die Gouvernementalisierung der Verwaltung wie die exekutive Inklination der Gouvernementalität glei-

45 An den Verhandlungen sind beteiligt die USA, Kanada, die EU, Australien, Japan, Südkorea, Mexiko, Neuseeland, Singapur, Marokko und die Schweiz. 
chermaßen. Die oben ausgewiesenen Kongruenzen zwischen Gouvernementalität und exekutiver Logik spiegeln sich in den Beispielen, die allesamt (1) einen Fokus im Bereich des Konkreten und des Partikularen haben, (2) dem Gesetz eine nachgelagerte, instrumentelle Bedeutung zuschreiben, (3) einen ordnungspolitischen Schwerpunkt aufweisen, (4) sich vornehmlich in technokratischen Steuerungs- und Managementmodi realisieren, (5) einen instrumentellen Erledigungs- und Problemlösungsbias vorweisen und schließlich (6) grenzüberschreitenden Sicherheitsfragen besondere Aufmerksamkeit zukommen lassen. Dabei kann die Einsicht in die staatstheoretisch-exekutive Inklination der Gouvernementalität insbesondere zum Verständnis des Phänomens beitragen, dass sich die gouvernementale Vernunft im Sicherheitsbereich in einem solchen Ausmaß institutionell, repressiv und illiberal realisiert, das der Gouvernementalität alleine nicht entspräche. Wenn dadurch keineswegs Foucaults Fokus auf die »Gouvernementalisierung des Staates« (2004a: 163, Hervorh. dort) zurückgenommen wird, kann doch die Anerkenntnis der exekutiven Einfärbung der gouvernementalen Vernunft nicht nur zu erklären helfen, wie der Staat gouvernementalisiert wird, sondern zugleich, weshalb diese Gouvernementalisierung sich zum einen in einem verwaltungsmäßigen mainstreaming realisiert und zum anderen in institutionalisierten Exekutivorganen einen besonders fruchtbaren Boden vorfindet. Kurzum: Wenn beispielsweise William Walters und Jens Henrik Haahr mit Blick auf die Europäische Union betonen, dass Gouvernementalisierung »does entail the construction of political and administrative structures« (2005: 139), macht eine exekutiv-gouvernementale Perspektive verständlich, weshalb das Ergebnis nun ausgerechnet in Frontex, einem zugleich biopolitischen Akteur und exekutiven Grenzschutzorgan besteht. Während die Gouvernementalität den Blick auf biopolitische Normalisierung von Subjekten und Bevölkerungen eröffnet, erweitert die Einsicht ihrer exekutiven Inklination die Perspektive über einen subjektologisch-biopolitischen Fokus hinaus.

Unter analytischen Gesichtspunkten ist die Rückbeziehung der Gouvernementalität auf die Exekutive in sicherheitspolitischen Studien somit nicht zuletzt deshalb von Belang, weil sie zur Erfassung der Gleichzeitigkeit von subjektfokussierter Gouvernementalität und traditioneller Exekutivpolitik in den grenzüberschreitenden Beziehungen beitragen, gleichsam die Transformation und Persistenz klassischer Sicherheitspolitiken ins Auge fassen kann. Eine solche integrative Perspektive vermag z.B. mit Blick auf bewaffnete Konflikte die »military transformation in the age of life as information« (Dillon/Reid 2009: 106) zu erkennen, durch die im Namen der »biohumanity« (Dillon/Reid 2009: 20) liberale Kriege geführt werden (vgl. Dillon/Reid 2009: 17-21, 34-38, 127-131), ohne aber die Persistenz klassischer internationaler und geostrategischer, oft keineswegs auf biopolitische Subjekte fokussierter Militärpolitiken analytisch aus dem Auge zu verlieren. Dies trägt auch dazu bei, der Gefahr eines Western bzw. European bias oder gar eines methodologischen Ethnozentrismus in der empirischen Forschung zu entgehen, indem auch Weltregionen wieder ins Sichtfeld rücken können, deren grenzüberschreitende Beziehungen und Konflikte sowie Regierungs- und Sicherheitsrationalitäten stärker klassischen Mustern folgen 
als dies in Europa der Fall ist. ${ }^{46}$ Ein exekutiv-gouvernementaler Zugang vermag klassisch-exekutive Dynamiken zu erfassen, wo gouvernementale Rationalitäten vorhanden, aber nicht dominant sind, und sich gleichsam stufenlos im Kontinuum zwischen gouvernementaler Logik und klassischer Regierungspolitik zu bewegen. Ohne in theoretischer Hinsicht eine staatstheoretisch-exekutive und eine gouvernementale Ebene wieder trennen oder gar gegeneinander ausspielen zu müssen, kann eine solche integrale Perspektive beispielsweise berücksichtigen, dass regionale Sicherheitsfragen etwa in Asien (vgl. Calder/Fukuyama 2008; Ikenberry/Mastanduno 2003) neben gouvernementalen Rationalitäten weiterhin stark von Logiken der great power politics gekennzeichnet sind.

Wir können es bei diesen Hinweisen auf die empirische Eignung und mögliche Ergiebigkeit eines exekutiv-gouvernementalen Theorierahmens belassen. Dabei gilt es, gleichsam als Disclaimer, hervorzuheben, dass aus der Diskussion und Kritik der exekutiv-gouvernementalen Entgrenzung der Sicherheitspolitik keinesfalls die Geringschätzung grenzüberschreitender Probleme und Gefahren oder gar die Forderung nach einer Renationalisierung politischer Strukturen resultiert. Auch wird damit keineswegs die Notwendigkeit bestritten, verschiedene, auch private Akteurstypen in Sicherheitsfragen (i.w.S.) einzubeziehen oder sicherheitsbezogene Politiken und $\mathrm{Maßnahmen} \mathrm{zu} \mathrm{entwerfen,} \mathrm{die} \mathrm{auch} \mathrm{Individuen} \mathrm{direkt} \mathrm{adressieren.} \mathrm{Zur} \mathrm{Debatte} \mathrm{steht}$ aber in der Tat die spezielle, exekutiv-gouvernementale Form der Sicherheit, d.h. diese besondere Rationalität der verwaltungsförmigen Entgrenzung politischer Strukturen, Dynamiken und Geltungsräume. Anhand der explorativen Diskussion einzelner Beispiele sollte gezeigt werden, dass und wie ein auf Foucaults Gouvernementalität zurückgehender, allerdings zugleich auf den Staatsapparat - und nicht lediglich auf ein allgemeines Korrelat der Souveränität, der souveränen Macht oder der Biopolitik - bezogener Ansatz empirisch konkretisierbar und operationalisierbar ist und insbesondere hinsichtlich von Fragen der grenzüberschreitenden Sicherheit eine fruchtbare Perspektive zu eröffnen verspricht. So erlaubt ein solcher theoretischer Rahmen einerseits den Ausweis und die Beschreibung von Kongruenzen und Zusammenhängen in zunächst unverbundenen policy-Feldern (Gesundheits-, Kriminalitäts-, Militär-, Migrations-, Drogen-, Lebensmittel- und Verbraucherschutzpolitik, etc.) sowie andererseits die Berücksichtigung der fortgesetzten Möglichkeit klassischer Sicherheitspolitiken sowie ihrer Integration in neue policy-Bereiche. Im Sinne der von Bob Jessop angemahnten »bridge between micro-diversity and macro-necessity« (2007: 39; vgl. 2011) ermöglicht eine integrative exekutiv-gouvernementale Sicht die Berücksichtigung der biopolitischen Mikro-, aber eben auch der formalen und institutionellen Makroebene aktueller (sicherheits-)politischer Strukturen und Dynamiken. Dabei kann sie schließlich die analytische Grundlage für eine Kritik exekutiver Anmaßungen bilden, deren Besonderheit just in der Gleichzeitigkeit von institutioneller Fundierung und Entgrenzung besteht.

46 Dass und inwiefern die Europäische Integration auch einer gouvernementalen politischen Rationalität verpflichtet ist, haben Walters (2004) sowie Walters/Haahr (2005) herausgearbeitet. 


\section{Schlussbemerkung}

Im vorliegenden Beitrag ist erstens die Plausibilität und Notwendigkeit einer theoretischen Kopplung des Gouvernementalitätsansatzes mit der staatstheoretischen Logik der Regierung ausgewiesen, zweitens die Korrespondenz einer solchen Perspektive insbesondere mit Strukturen und Dynamiken inter-, trans- und supranationaler Politik demonstriert sowie drittens dieser theoretisch begründete Zugang empirisch exemplifiziert worden. Die hier unternommenen Ausführungen können als ein konkreter Vorschlag gelten, wie das analytische und kritische Potenzial der Foucaultschen Gouvernementalitätstheorie in den IB auch jenseits Foucaultscher Spezialdiskurse fruchtbar gemacht werden kann, ohne dass es einerseits in einem solchen Maße verwässert wird, dass es sich in einer Integration von poststrukturalistischen Gemeinplätzen (Diskurse sind wichtig, politische Tatbestände sind sozial konstruiert, etc.) erschöpft, oder andererseits im Sinne einer Globaltheorie einen empirischen Zugriff letztlich ausschließt. ${ }^{47}$

Gleichwohl ist einschränkend darauf hinzuweisen, dass die exemplifizierende Diskussion in Abschnitt 4 freilich nicht nur in empirischer Hinsicht nicht erschöpfend ist, sondern ebenso in systematischer Hinsicht fortzuführen wäre. So würde eine auf exekutive Gouvernementalisierung fokussierte Analyse sich detailliert mit der Struktur, den Akteuren und nicht zuletzt auch den Interessenskonflikten und Widerständen innerhalb bestimmter Sicherheitsdiskurse, d.h. in gegenständlicher Eingrenzung auseinanderzusetzen haben. Auch müsste sie sich der Frage zuwenden, wie »die Identifikations- und Präventionstechniken unserer Gegenwart [...] die Schwelle operationalisieren, an der die liberale Regierung in den illiberalen Modus wechselt « (Opitz 2008: 218).

Die angestellten Überlegungen können gleichwohl als ein Beitrag zu einem Umgang mit Foucaultschen Ansätzen in den IB gelten, den Kevin Stenson als »realistische Gouvernementalitätstheorie « (Stenson 2007: 191, Hervorh. dort) bezeichnet und der »die vorherrschende Lesart diskursiver Gouvernementalitätsthorie« (Stenson 2007: 191, Hervorh. dort) herausfordert. Der Begriff des Realismus ist hier nicht im Sinne der Denkschule in den IB verwendet, sondern markiert die Notwendigkeit einer Rückbeziehung der Gouvernementalitätstheorie auf die materiale (institutionelle, rechtliche, gewaltförmige, etc.) Apparatur der Politik. Das bei Foucault vorliegende Desinteresse an staatlichen Institutionen und staatstheoretischen Konzepten, das in der Verwendung kumulativer Begriffe (die Souveränität, der König) als negative Korrelate zum Ausdruck kommt, sollte dabei nicht wiederholt und Foucault in seinem Anspruch einer Abwendung vom Staat nicht die Treue gehalten werden. Vielmehr gilt insbesondere mit Blick auf die staatstheoretische Reflexion des Foucaultschen Denkens, »dass die Gouvernementalität der Zukunft die Staatsphobie überwinden« (Heidenreich 2011b: 155) muss.

Einen solchen Weg der staatstheoretischen Rückbeziehung der Gouvernementalität zu erproben, scheint schließlich auch deshalb wichtig zu sein, um sich in der Unter-

47 Man könnte ersteres als IB-Gefahr, letzteres als Politische-Theorie-Gefahr bezeichnen. 
suchung inter-, trans- und supranationaler Politik nicht der kritischen Dynamik des gouvernementalen Ansatzes zu begeben. Denn wenn mit der exekutiven Inklination gleichsam der eben auch im heutigen Wortsinne durchgängig »polizeiliche Charakter der illiberalen Gouvernementalität« (Opitz 2008: 221, meine Hervorh.) sowohl theoretisch begründet als auch an Beispielen veranschaulicht werden konnte, dann wird damit ipso facto das kritische Potenzial eines solchen Ansatzes offensichtlich. Die Verwobenheit von Gouvernementalität und exekutiver Logik, die zum einen in der Ausdehnung von exekutiv-gouvernementalen Rationalitäten und Handlungsbereichen über die institutionellen, territorialen und geltungsräumlichen Grenzen der Verwaltung hinaus und zum anderen in der verbindlichen Realisierung und zwangsbewehrten Durchsetzung verwaltungsförmiger Politik in praxi besteht, wirft unmittelbar Fragen der Legitimität und der Rechtfertigung politischer Macht auf. Dabei substantiiert der explizite theoretische und analytische Rückbezug der Gouvernementalitätstheorie auf die Regierung (i.e.S.) die Kritik an einer allgemeinen politischen Rationalität, die sowohl grenzenlos entgrenzend als auch auf die administrativ-institutionelle Produktion greifbarer Ergebnisse ausgerichtet ist und dabei als verwaltungslogisches mainstreaming funktioniert. So kann eine staatstheoretisch fundierte Gouvernementalitätstheorie m.E. als genuin kritische Theorie gelten, die analytisch breit, aber empirisch konkretisierbar die Schattenseiten entgrenzter Politik als Verwaltung ausleuchten und die grenzüberschreitenden Selbstermächtigungen exekutiver Macht offenlegen kann.

\section{Literatur}

Agamben, Giorgio 2002: Homo sacer. Die souveräne Macht und das nackte Leben, Frankfurt a.M.

Albert, Mathias/Lenco, Peter 2008: Introduction to the Forum - Foucault and Political Sociology, in: International Political Sociology 3: 2, 265-266.

Amoore, Louise 2008: Foucault Against the Grain, in: International Political Sociology 3: 2, 274-276.

Anter, Andreas 2003: Im Schatten des Leviathan - Staatlichkeit als Ordnungsidee und Ordnungsinstrument, in: Bendel, Petra/Croissant, Aurel/Rüb, Friedbert W. (Hrsg.) unter Mitarbeit von Timo Freudenberger: Demokratie und Staatlichkeit. Systemwechsel zwischen Staatsreform und Staatskollaps, Opladen, 35-55.

Aradau, Claudia 2004: The Perverse Politics of Four-Letter Words: Risk and Pity in the Securitisation of Human Trafficking, in: Millennium - Journal of International Studies 33: 2, 251-277.

Aradau, Claudia/van Munster, Rens 2007: Governing Terrorism Through Risk: Taking Precautions, (Un)Knowing the Future, in: European Journal of International Relations 13: 1, 89-115.

Barry, Andrew/Osborne, Thomas/Rose, Nikolas (Hrsg.) 1996: Foucault and Political Reason: Liberalism, Neo-Liberalism and Rationalities of Government, Chicago, IL.

Bedorf, Thomas/Röttgers, Kurt (Hrsg.) 2010: Das Politische und die Politik, Berlin.

Bigo, Didier 2008: Security: A Field Left Fallow, in: Dillon, Michael/Neal, Andrew W. (Hrsg.): Foucault on Politics, Security and War, Houndmills, 93-114. 
Bigo, Didier/Tsoukala, Anastassia 2008: Understanding (In)Security, in: Bigo, Didier/Tsoukala, Anastassia (Hrsg.): Terror, Insecurity and Liberty: Illiberal Practices of Liberal Regimes after 9/11, Abingdon, 1-9.

Bigo, Didier/Walker, R.B.J./Chercheurs ELISE 2006: Liberté et Sécurité en Europe. Enjeux contemporains, in: Cultures \& Conflits 61: XX, 103-136.

Booth, Ken (Hrsg.) 2005: Critical Security Studies and World Politics, Boulder, CO.

Brauch, Hans Günter/Chadha Behera, Navnita/Kameri-Mbote, Patricia/Grin, John/Oswald Spring, Úrsula/Chourou, Béchir/Mesjasz, Czeslaw/Krummenacher, Heinz (Hrsg.) 2009: Facing Global Environmental Change: Environmental, Human, Energy, Food, Health and Water Security Concepts, New York, NY.

Bröckling, Ulrich/Krasmann, Susanne/Lemke, Thomas (Hrsg.) 2000: Gouvernementalität der Gegenwart. Studien zur Ökonomisierung des Sozialen, Frankfurt a.M.

Bröckling, Ulrich/Krasmann, Susanne/Lemke, Thomas (Hrsg.) 2011: Governmentality: Current Issues and Future Challenges, New York, NY.

Burchell, Graham/Gordon, Colin/Miller, Peter (Hrsg.) 1991: The Foucault Effect: Studies in Governmentality, Chicago, IL.

Butler, Judith 2003: Noch einmal. Körper und Macht, in: Honneth, Axel/Saar, Martin (Hrsg.): Michel Foucault. Zwischenbilanz einer Rezeption, Frankfurt a.M., 52-67.

Buzan, Barry/Hansen, Lene 2009: The Evolution of International Security Studies, Cambridge.

Buzan, Barry/Waever, Ole/de Wilde, Jaap 1998: Security: A New Framework for Analysis, Boulder, CO.

Bundesverfassungsgericht 2001: Leitsätze zum Urteil des Zweiten Senats vom 22. November 2001, 2 BvE 6/99, 22.11.2001, in: http://www.bverfg.de/entscheidungen/es20011122_2bve000699.html; 09.09.2011.

C.A.S.E. Collective 2006: Critical Approaches to Security in Europe: A Networked Manifesto, in: Security Dialogue 37: 4, 443-487.

Calder, Kent E./Fukuyama, Francis (Hrsg.) 2008: East Asian Multilateralism: Prospects for Regional Stability, Baltimore, MD.

Caldwell, Anne 2007: Die Regierung der Menschheit. Gouvernementalität und Bio-Souveränität, in: Krasmann/Volkmer 2007, 107-126.

Castel, Robert 1991: From Dangerousness to Risk, in: Burchell et al. 1991, 281-298.

Cerny, Philip G. 2010: Rethinking World Politics: A Theory of Transnational Neopluralism, Oxford.

Commission of the European Communities 2005: Proposal for a Regulation of the European Parliament and the Council on Common Rules in the Field of Civil Aviation Security (Text with EEA relevance), Brüssel, 03.10.2005.

Council of the European Union 2009: Council Conclusions on Alcohol and Health: 2980th Employment, Social Policy, Health and Consumer Affairs Council Meeting, Brüssel, 01.12.2009.

Czempiel, Ernst-Otto 1999: Kluge Macht. Außenpolitik für das 21. Jahrhundert, München.

Daase, Christopher/Kessler, Oliver 2007: Knowns and Unknowns in the »War on Terror«: Uncertainty and the Political Construction of Danger, in: Security Dialogue 38: 4, 411-436.

Dean, Mitchell 1999: Governmentality: Power and Rule in Modern Society, London.

Dean, Mitchell 2007: Governing Societies: Political Perspectives on Domestic and International Rule, Maidenhead.

de Larrinaga, Miguel/Doucet, Marc G. 2008: Sovereign Power and the Biopolitics of Human Security, in: Security Dialogue 39: 5, 517-537.

de Larrinaga, Miguel/Doucet, Marc G. (Hrsg.) 2010a: Security and Global Governmentality: Globalization, Governance and the State, Abingdon.

de Larrinaga, Miguel/Doucet, Marc G. 2010b: Governmentality, Sovereign Power and Intervention: Security Council Resolutions and the Invasion of Iraq, in: de Larrinaga/Doucet 2010a, 96-110. 
de Montesquieu, Charles 1992: Vom Geist der Gesetze, Band 1, übers. u. hrsg. v. Ernst Forsthoff, Tübingen.

Demirović, Alex 2008: Liberale Freiheit und das Sicherheitsdispositiv. Der Beitrag von Michel Foucault, in: Purtschert, Patricia/Meyer, Katrin/Winter, Yves (Hrsg.): Gouvernementalität und Sicherheit. Zeitdiagnostische Beiträge im Anschluss an Foucault, Bielefeld, 229-250.

Diez, Thomas 1999: Speaking »Europe«: The Politics of Integration Discourse, in: Journal of European Public Policy 6: 4, 598-613.

Diez, Thomas 2008: Michel Foucault and the Problematization of European Governance, in: International Political Sociology 3: 2, 266-268.

Dillon, Michael 2004: The Security of Governance, in: Larner, Wendy/Walters, William (Hrsg.): Global Governmentality: Governing International Spaces, London, 76-94.

Dillon, Michael 2007: Governing Terror: The State of Emergency of Biopolitical Emergence, in: International Political Sociology 1: 1, 7-28.

Dillon, Michael/Neal, Andrew W. (Hrsg.) 2008: Foucault on Politics, Security and War, Houndmills.

Dillon, Michael/Reid, Julian 2009: The Liberal Way of War: Killing to Make Life Live, London.

Di Muzio, Tim 2008: Governing Global Slums: The Biopolitics of Target 11, in: Global Governance 14: 3, 305-326.

Ferguson, James/Gupta, Akhil 2002: Spatializing States: Toward an Ethnography of Neoliberal Governmentality, in: American Ethnologist 29: 4, 981-1002.

Food and Agriculture Organization of the United Nations (FAO) 1996: Rome Declaration on World Food Security: World Food Summit, 13-17 November 1996, Rome/Italy, in: http:// www.fao.org/DOCREP/003/W3613E/W3613E00.HTM; 12.08.2010.

Foucault, Michel 1975: Surveiller et punir. Naissance de la prison, Paris.

Foucault, Michel 1976: Mikrophysik der Macht. Über Strafjustiz, Psychiatrie und Medizin, Berlin.

Foucault, Michel 1978a: Recht der Souveränität/Mechanismus der Disziplin. Vorlesung vom 14. Januar 1976, in: Foucault, Michel: Dispositive der Macht. Über Sexualität, Wissen und Wahrheit, Berlin, 75-95.

Foucault, Michel 1978b: Wahrheit und Macht. Interview von A. Fontana und P. Pasquino, in: Foucault, Michel: Dispositive der Macht. Über Sexualität, Wissen und Wahrheit, Berlin, 21-54.

Foucault, Michel 1994: Das Subjekt und die Macht, in: Dreifuß, Hubert L./Rabinow, Paul: Michel Foucault. Jenseits von Strukturalismus und Hermeneutik, 2. Auflage, Weinheim, 241-261.

Foucault, Michel 1996: Diskurs und Wahrheit. Die Problematisierung der Parrhesia, Berlin.

Foucault, Michel 1998: Der Wille zum Wissen. Sexualität und Wahrheit I, 10. Auflage, Frankfurt a.M.

Foucault, Michel 2000a: Die »Gouvernementalität«, in: Bröckling et al. 2000, 41-67.

Foucault, Michel 2000b: Staatsphobie, in: Bröckling et al. 2000, 68-71.

Foucault, Michel 2004a: Geschichte der Gouvernementalität I. Sicherheit, Territorium, Bevölkerung. Vorlesung am Collège de France 1977-1978, Frankfurt a.M.

Foucault, Michel 2004b: Geschichte der Gouvernementalität II. Die Geburt der Biopolitik. Vorlesung am Collège de France 1978-1979, Frankfurt a.M.

Fougner, Tore 2008: Neoliberal Governance of States: The Role of Competitiveness Indexing and Country Benchmarking, in: Millennium - Journal of International Studies 37: 2, 303326.

Frankfurter Rundschau 14./15.08.2010: Geheimsache Zukunft. Ab Montag wird über das ActaAbkommen verhandelt. Bürgerrechtler warnen, es verstoße gegen Menschenrechte, 6-7.

Fraser, Nancy 2003: Von der Disziplin zur Flexibilisierung? Foucault im Spiegel der Globalisierung, in: Honneth, Axel/Saar, Martin (Hrsg.): Michel Foucault. Zwischenbilanz einer Rezeption, Frankfurt a.M., 239-258. 
Friedman, Jonathan/Randeria, Shalini 2004: Worlds on the Move: Globalisation, Migration and Cultural Security, London.

Garland, David 2002: The Culture of Control: Crime and Social Order in Contemporary Society, Oxford.

Gehring, Petra 2004: Foucault - Die Philosophie im Archiv, Frankfurt a.M.

Habermas, Jürgen 1998: Die postnationale Konstellation. Politische Essays, Frankfurt a.M.

Heidenreich, Felix (Hrsg.) 2011a: Technologien der Macht. Zu Michel Foucaults Staatsverständnis, Baden-Baden.

Heidenreich, Felix 2011b: Foucaults Rekonstruktion des Liberalismus und die Rolle des Staates, in: Heidenreich 2011a, 139-155.

Held, David 2007: Soziale Demokratie im globalen Zeitalter, Frankfurt a.M.

Heller, Hermann 1971: Recht, Staat, Macht. Gesammelte Schriften, Band 2, Leiden.

Heng, Yee-Kuang/McDonagh, Ken 2008: The Other War on Terror Revealed: Global Governmentality and the Financial Action Task Force's Campaign against Terrorist Financing, in: Review of International Studies 34: 3, 553-573.

Hindess, Barry 1996: Discourses of Power: From Hobbes to Foucault, Oxford.

Hindess, Barry 2008: Government and Discipline, in: International Political Sociology 3: 2, 268-270.

Hobbes, Thomas 1998: Leviathan oder Stoff, Form und Gewalt eines kirchlichen und bürgerlichen Staates. Hrsg. und eingel. von Iring Fetscher, übers. von Walter Euchner, 8. Auflage, Frankfurt a.M.

Ikenberry, G. John/Mastanduno, Michael (Hrsg.) 2003: International Relations Theory and the Asia-Pacific, New York, NY.

Jabri, Vivienne 2006: War, Security and the Liberal State, in: Security Dialogue 37: 1, 47-64.

Jellinek, Georg 1966: Allgemeine Staatslehre, 3. Auflage, Bad Homburg v.d.H.

Jessop, Bob 2007: From Micro-Powers to Governmentality: Foucault's Work on Statehood, State Formation, Statecraft and State Power, in: Political Geography 26: 1, 34-40.

Jessop, Bob 2011: Constituting Another Foucault Effect: Foucault on States and Statecraft, in: Bröckling et al. 2011, 56-73.

Jones, Bruce 2006: Bio-Security, Nonstate Actors and the Need for Global Cooperation, in: Ethics \& International Affairs 20: 2, 225-228.

Joseph, Jonathan 2010: The Limits of Governmentality. Social Theory and the International, in: European Journal of International Relations 16: 2, 223-246.

Joseph, Jonathan 2011: Governmentality of What? Populations, States and International Organisations, in: Kiersey, Nicholas J./Stokes, Doug (Hrsg.): Foucault and International Relations: New Critical Engagements, Abingdon, 51-65.

Kammler, Clemens 1986: Michel Foucault. Eine kritische Analyse seines Werks, Bonn.

Keohane, Robert O. 2006: The Contingent Legitimacy of Multilateralism, in: GARNET Working Paper 09/06.

Kerchner, Brigitte 2006: Wirklich Gegendenken. Politik analysieren mit Michel Foucault, in: Kerchner, Brigitte/Schneider, Silke (Hrsg.): Foucault. Diskursanalyse der Politik. Eine Einführung, Wiesbaden, 145-164.

Kerchner, Brigitte/Schneider, Silke 2006: »Endlich Ordnung in der Werkzeugkiste«. Zum Potenzial der Foucaultschen Diskursanalyse für die Politikwissenschaft - Einleitung, in: Kerchner, Brigitte/Schneider, Silke (Hrsg.): Foucault. Diskursanalyse der Politik. Eine Einführung, Wiesbaden, 9-30.

Kiersey, Nicholas J. 2010: Neoliberal Political Economy and the Iraq War: A Contribution to the Debate about Global Biopolitics, in: de Larrinaga/Doucet 2010a, 61-78.

Krasmann, Susanne/Volkmer, Michael (Hrsg.) 2007: Michel Foucaults »Geschichte der Gouvernementalität « in den Sozialwissenschaften. Internationale Beiträge, Bielefeld.

Larner, Wendy/Walters, William 2004: Globalization as Governmentality, in: Alternatives 29: $5,495-514$. 
Lembcke, Oliver W. 2010: Konstitutionelles Gesetz im sozialen Rechtsstaat. Zu einem Grundbegriff der Staatslehre Hellers, in: Llanque, Marcus (Hrsg.): Souveräne Demokratie und soziale Homogenität. Das politische Denken Herrmann Hellers, Baden-Baden, 239-260.

Lemke, Thomas 1997: Eine Kritik der politischen Vernunft. Foucaults Analyse der modernen Gouvernementalität, Berlin.

Lemke, Thomas 2007: Eine unverdauliche Mahlzeit? Staatlichkeit, Wissen und die Analytik der Regierung, in: Krasmann/Volkmer 2007, 47-73.

Lentzos, Filippa/Rose, Nikolas 2008: Die Unsicherheit regieren. Biologische Bedrohungen, Notfallplanung, Schutz und Resilienz in Europa, in: Purtschert, Patricia/Meyer, Katrin/ Winter, Yves (Hrsg.): Gouvernementalität und Sicherheit. Zeitdiagnostische Beiträge im Anschluss an Foucault, Bielefeld, 75-101.

Linklater, Andrew 2005: Political Community and Human Security, in: Booth 2005, 113-131.

Lipschutz, Ronnie D./Rowe, James K. 2005: Globalization, Governmentality and Global Politics: Regulating for the Rest of Us?, Abingdon.

Locke, John 1966: Two Treatises of Government: A Critical Edition with an Introduction and Apparatus Criticus by Peter Laslett, Cambridge.

Merlingen, Michael 2008: Monster Studies, in: International Political Sociology 3: 2, 272-274.

Moran, Daniel/Russell, James A. (Hrsg.) 2009: Energy Security and Global Politics: The Militarization of Resource Management, Abingdon.

Mouffe, Chantal 2005: On the Political, London.

Müller, Harald 2009: Staatlichkeit ohne Staat - ein Irrtum aus der europäischen Provinz? Limitierende Bedingungen von Global Governance in einer fragmentierten Welt, in: Deitelhoff, Nicole/Steffek, Jens (Hrsg.): Was bleibt vom Staat? Demokratie, Recht und Verfassung im globalen Zeitalter, Frankfurt a.M., 221-258.

Müller, Klaus 2002: Globalisierung, Frankfurt a.M.

Neumann, Iver B./Sending, Ole Jacob 2007: »The International« as Governmentality, in: Millennium - Journal of International Studies 35: 3, 677-701.

Opitz, Sven 2004: Gouvernementalität im Postfordismus. Macht, Wissen und Techniken des Selbst im Feld unternehmerischer Rationalität, Hamburg.

Opitz, Sven 2008: Zwischen Sicherheitsdispositiven und Securitization. Zur Analytik illiberaler Gouvernementalität, in: Purtschert, Patricia/Meyer, Katrin/Winter, Yves (Hrsg.): Gouvernementalität und Sicherheit. Zeitdiagnostische Beiträge im Anschluss an Foucault, Bielefeld, 201-228.

Risse, Thomas 2004: Social Constructivism and European Integration, in: Wiener, Antje/Diez, Thomas (Hrsg.): European Integration Theory, Oxford, 159-176.

Rorty, Richard 1992: Kontingenz, Ironie und Solidarität, Frankfurt a.M.

Rosa, Hartmut 2005: Beschleunigung. Die Veränderung der Zeitstrukturen in der Moderne, Frankfurt a.M.

Rousseau, Jean-Jacques 1977: Vom Gesellschaftsvertrag oder Grundsätze des Staatsrechts. In Zusammenarbeit mit Eva Pietzcker neu übersetzt und herausgegeben von Hans Brockard, Stuttgart.

Schmitt, Carl 1996: Die geistesgeschichtliche Lage des heutigen Parlamentarismus, 8. Auflage, Berlin.

Schuck, Christoph (Hrsg.) 2011: Security in a Changing Global Environment: Challenging the Human Security Approach, Baden-Baden.

Simons, Jon 1995: Foucault \& the Political, London.

Stenson, Kevin 2007: Staatsmacht, Biopolitik und die lokale Regierung von Kriminalität in Großbritannien, in: Krasmann/Volkmer 2007, 181-209.

Süddeutsche Zeitung 26.12.2009: USA: Vereitelter Anschlag in Flugzeug. Verschärfte Kontrollen, in: http://www.sueddeutsche.de/politik/usa-vereitelter-anschlag-in-flugzeug-verschaerfte-kontrollen-1.76879; 02.08.2011.

Traub-Werner, Marion 2007: Free Trade: A Governmentality Approach, in: Environment and Planning A 39: 6, 1441-1456. 
United Nations Development Programme (UNDP) 1994: Human Development Report 1994, New York, NY.

Vasilache, Andreas 2007: Der Staat und seine Grenzen. Zur Logik politischer Ordnung, Frankfurt a.M.

Vasilache, Andreas 2009: The Normative and Analytical Limits of Governance: A Conceptual Intervention and a Poststructuralist Suggestion, in: de Sales Marques, José Luis/Seidelmann, Reimund/Vasilache, Andreas (Hrsg.): Asia and Europe: Dynamics of Inter- and Intra-Regional Dialogues, Baden-Baden, 59-77.

Vasilache, Andreas 2011a: Human Securitization: State Theory, Governmentality and the Ambivalence of Security in Europe, in: Schuck 2011, 123-152.

Vasilache, Andreas 2011b: The Rise of Executive Sovereignty in the Era of Globalization, in: Telò, Mario (Hrsg.): The State, Globalization and Multilateralism: Continuity and Change, New York, NY, i.E.

Wever, Ole 2004: Discursive Approaches, in: Wiener, Antje/Diez, Thomas (Hrsg.): European Integration Theory, Oxford, 197-215.

Walters, William 2004: The Political Rationality of European Integration, in: Larner, Wendy/ Walters, William (Hrsg.): Global Governmentality: Governing International Spaces, London, 155-173.

Walters, William/Haahr, Jens Henrik 2005: Governing Europe: Discourse, Governmentality and European Integration, London.

Weber, Max 1947: Schriften zur theoretischen Soziologie, zur Soziologie der Politik und Verfassung, Frankfurt a.M.

Wendt, Alexander 2004: Social Theory of International Politics, 7. Auflage, Cambridge.

Wiener, Antje 2003: Constructivism: The Limits of Bridging Gaps, in: Journal of International Relations and Development 6: 2, 253-276.

Winter, Yves 2008: Gewaltökonomien und Unsicherheit. Zur Gouvernementalität der »neuen Kriege«, in: Purtschert, Patricia/Meyer, Katrin/Winter, Yves (Hrsg.): Gouvernementalität und Sicherheit. Zeitdiagnostische Beiträge im Anschluss an Foucault, Bielefeld, 49-74.

Wolf, Klaus-Dieter 1999: The New Raison d'État as a Problem for Democracy in World Society, in: European Journal of International Relations 5: 3, 333-363.

World Health Organisation (WHO) 2007: A Safer Future: Global Public Health Security in the 21st Century: The World Health Report 2007, Genf.

World Health Organization (WHO) 2008: WHO Policy on Non-Recruitment of Smokers or other Tobacco Users: Frequently Asked Questions, Genf.

Zehfuss, Maja 2002: Constructivism in International Relations: The Politics of Reality, Cambridge.

Zeitschrift für Internationale Beziehungen 2010: Symposium Internationale Politische Theorie, in: Zeitschrift für Internationale Beziehungen 17: 2, 267-363. 